\title{
X-Ray Powder Data
}

For Uranium And

Thorium Minerals

GEOLOGICAL SURVEY BULLETIN 1036-G

This report concerns work done on behalf of the U. S. Atomic Energy Commission and is published with the permission of the Commission

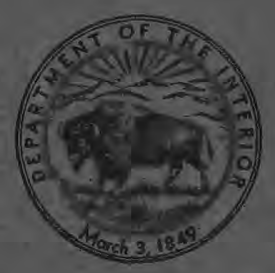




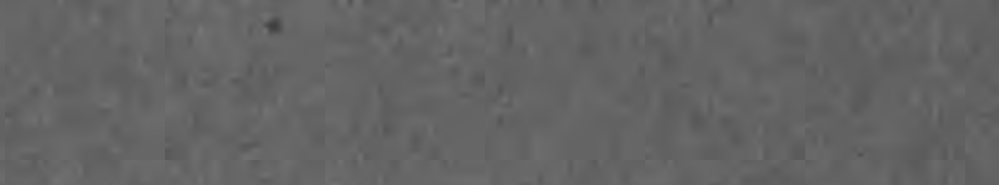
94.

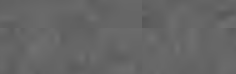

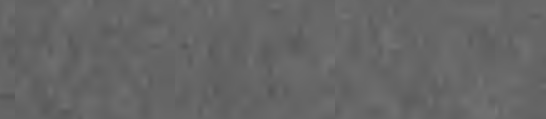

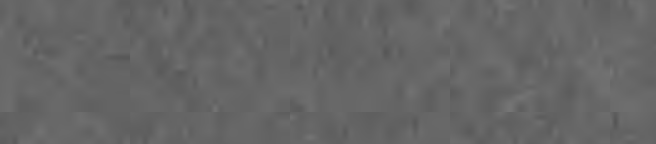

atitis

$+1$.

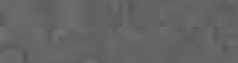

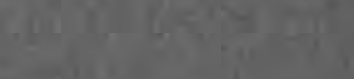

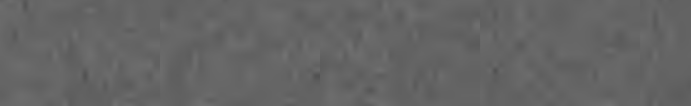

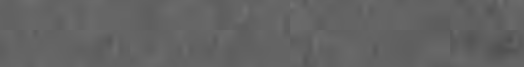

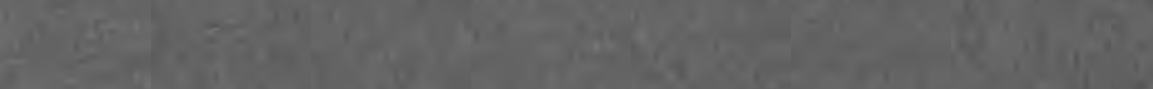

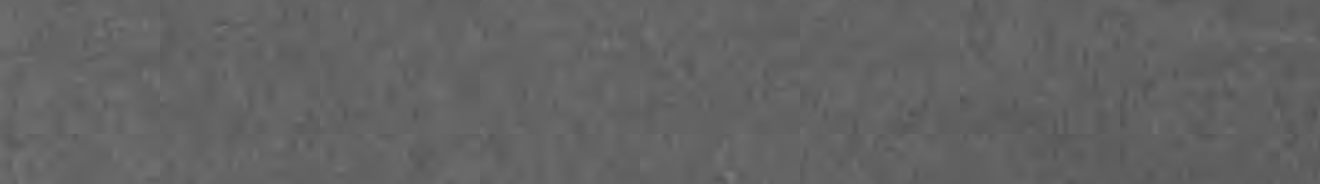

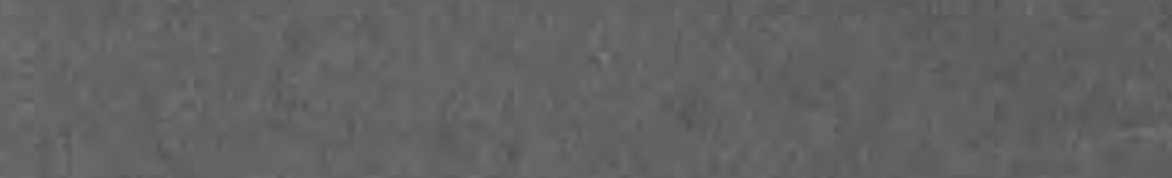
$x^{2}+2=$
1)

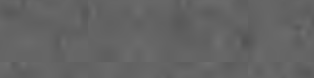

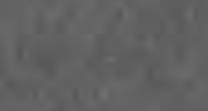

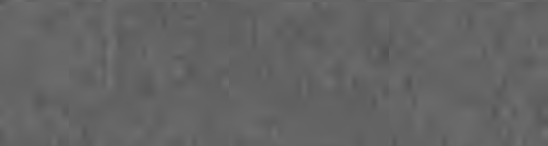

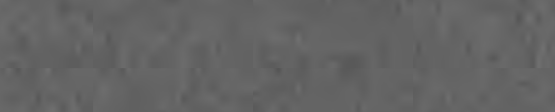

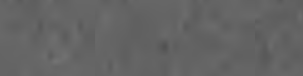

$\operatorname{lin}^{2}+\frac{1}{2}+3$

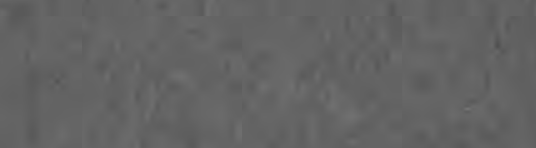

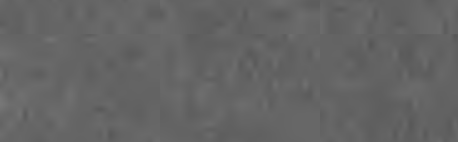

\section{(1)}
5

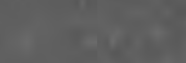

$y+\frac{10}{20}$

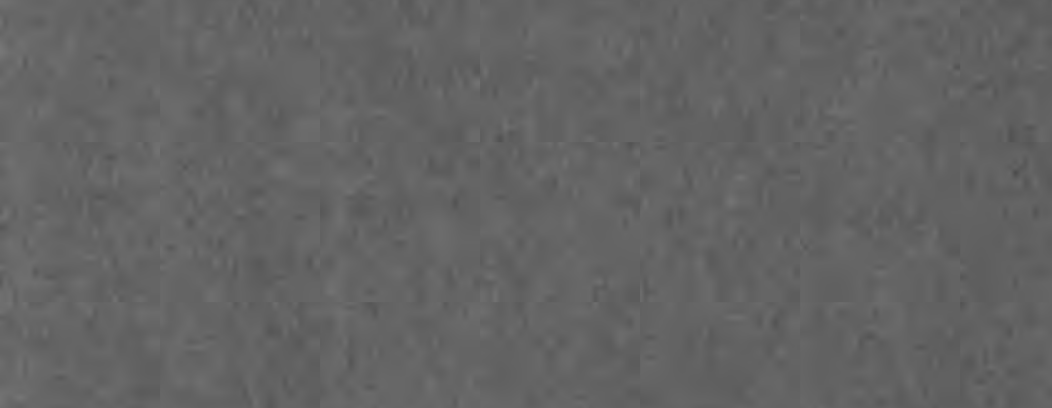

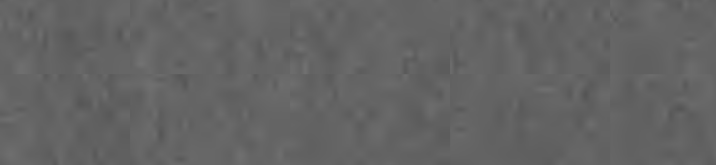

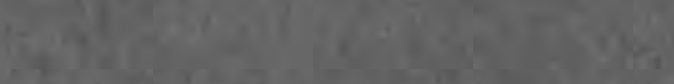

16

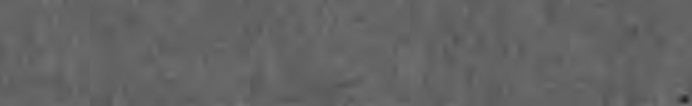
if. 


\section{X-Ray Powder Data}

For Uranium And

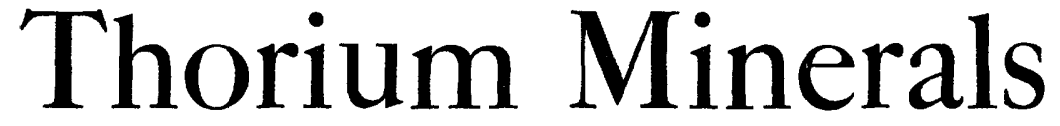

By CLIFFORD FRONDEL, DAPHNE RISKA, and JUDITH WEISS FRONDEL

A CONTRIBUTION TO GEOCHEMISTRY

GE OLOGICAL SURVEY B U L L E T I N 1036-G

This report concerns work done on behalf of the U.S. Atomic Energy Commission and is published with the permission of the Commission

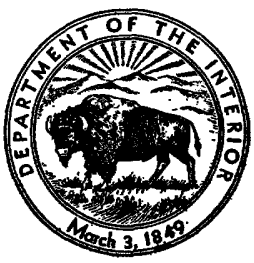


UNITED STATES DEPARTMENT OF THE INTERIOR

Fred A. Seaton, Secretary

\section{GEOLOGIGAL SURVEY}

Thomas B. Nolan, Director

For sale by the Superintendent of Documents, U. S. Government Printing Office 


\section{CONTENTS}

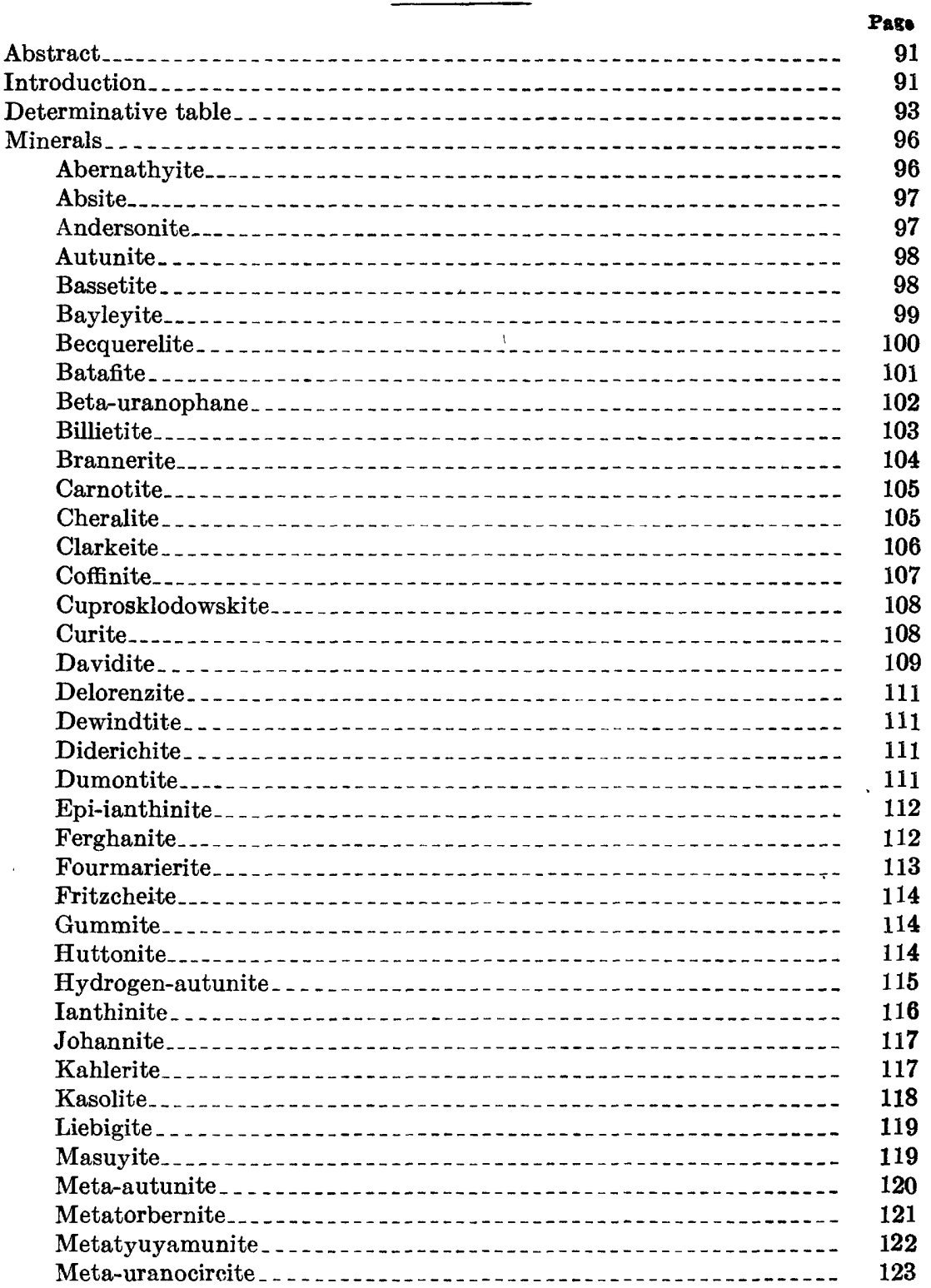


Minerals-Continued Page

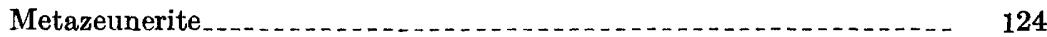

Microlite-pyrochlore . . .

Novacekite . . . . . .

Parsonsite . . .

Peligotite

Phosphuranylite

Pilbarite

Rabbittite

Rauvite....

Renardite.

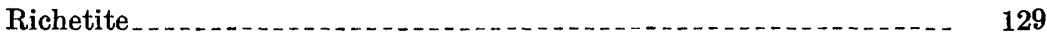

Rutherfordine_...

Sabugalite...

Saléeite . . . .

Samiresite .

Schoepite $\ldots \ldots \ldots$

Schroeckingerite

Sengierite $\ldots \ldots \ldots$

Sharpite

Sklodowskite .....

Soddyite _. . . .

Studtite $\ldots \ldots \ldots \ldots$

Swartzite $\ldots \ldots \ldots \ldots \ldots$

Thorianite . . . . .

Thorite

Thorogummite _...

Torbernite............... 138

Troegerite ................. 139

Tyuyamunite............. 140

Umohoite

Uraninite ...

Uranophane . . .

Uranopilite . . . . . . . . . . . .

Uranosphathite

Uranospherite

Uranospinite...... 145

Uvanite ...

Vandenbrandeite . . .

Vandendriesscheite . . . . . . .

Voglite

Walpurgite $\ldots \ldots$

Zeunerite

Zippeite

References............ 152 


\section{A CONTRIBUTION TO GEOCHEMISTRY}

\section{X-RAY POWDER DATA FOR URANIUM AND THORIUM MINERALS}

By Clifford Frondel, Daphne Riska, and Judith Weiss Frondel

\section{ABSTRACT}

X-ray powder spacing data are given for 67 uranium and thorium minerals. The spacing data and line intensities are given for each mineral and also in the form of a determinative table based on the three most intense lines for each mineral arranged in order of decreasing spacing.

\section{INTRODUCTION}

The U. S. Geological Survey has in preparation a comprehensive volume on the mineralogy of uranium and thorium. This work has been done as part of a continuing systematic survey of data on uranium and thorium minerals on behalf of the Division of Raw Materials, U. S. Atomic Energy Commission.

Pending publication of this volume and in response to a widespread demand among workers in uranium and thorium mineralogy, the X-ray powder diffraction data for the known minerals that contain uranium or thorium as an essential constituent are presented here. The coverage is complete except for a few minerals for which there are no reliable data, owing to lack of authentic specimens.

With the exception of that for ianthinite, the new data originated either in the Geological Survey or in the Mineralogical Laboratory of Harvard University. Data from the literature or other sources were cross-checked against the files of standard patterns of these laboratories; the sources are indicated in the references. Data not accompanied by a reference were obtained from films in the Harvard Standard File and cross-checked as to the identity of the film with the Geological Survey's file. 
Minor differences can be expected in the $d$-spacings reported for the same specimens by different investigators because of the manner of preparation of the mount, the conditions of X-ray irradiation, and the method of photography and measurement of the film or chart. The Harvard and Geological Survey data all were obtained from films taken in 114-mm diameter cameras, using either ethyl cellulose and toluene or collodion spindle mounts and Straumanis-type film mounting. Unless otherwise indicated all patterns were taken with copper radiation $(\mathrm{K} \alpha=1.5418 \mathrm{~A})$ and nickel filter and data are given in angstrom units. The $d$-spacings are not corrected for film shrinkage. The correction ordinarily is small and in general is less than either the variation in spacing arising from differences in experimental technique of different investigators, including the varying absorption of samples of different thickness and concentration, or the variation attending slight changes in the chemical composition of the mineral.

Some uranium minerals give poor diffraction patterns. The best results are generally obtained by using relatively small-diameter spindles and long exposures, with a takeoff angle from the X-ray tube of about $4^{\circ}$. It is sometimes advantageous to shield the film from fluorescence in the visible region excited by X-ray irradiation. Copper radiation is preferable. The patterns of a few uranium minerals are greatly impaired by heavy grinding of the sample. Light crushing of the coarse sample after mixing with about one-third its volume of coarsely powdered low-absorption glass is helpful.

Many uranium minerals, such as the members of the torbernite group, readily lose zeolitic water or transform to lower hydrates at or near ordinary conditions of temperature and humidity and care should be taken to control this in the manner or preservation and preparation of the sample. 


\section{DETERMINATIVE TABLE}

The following table lists the uranium and thorium minerals in order of decreasing spacing of the most intense line present in the pattern. Two or more entries are made for minerals whose patterns contain two or more darkest lines of the same or nearly the same intensity. In addition, the three next darkest lines are listed in order of decreasing intensity for each species.

The cameras and radiation used are such that lines corresponding to $d$-spacings of greater than $17 \mathrm{~A}$ could not be recorded even if present. The error in line measurement is estimated at $\pm 0.1^{\circ} 2 \theta$ or an upper limit of error of $0.35 \mathrm{~A}$ for $d$-spacings in the range $5^{\circ}-10^{\circ}$ $2 \theta, 0.1 \mathrm{~A}$ in the range $10^{\circ}-30^{\circ}, 0.01 \mathrm{~A}$ in the range $30^{\circ}-90^{\circ}$, and less than $0.002 \mathrm{~A}$ for spacings with $2 \theta$ greater than $90^{\circ}$.

\begin{tabular}{|c|c|c|c|c|}
\hline $\begin{array}{l}\text { Darkest line } \\
\text { (angstrom) }\end{array}$ & Mineral & \multicolumn{3}{|c|}{$\begin{array}{l}\text { Three next darkest } \\
\text { lines (angstrom) }\end{array}$} \\
\hline $\begin{array}{l}\text { 13. } 0 \\
10.7 \\
10.33 \\
10.33 \\
10.3\end{array}$ & $\begin{array}{l}\text { Andersonite } \\
\text { Rauvite } \\
\text { Zeunerite } \\
\text { Autunite } \\
\text { Torbernite }\end{array}$ & $\begin{array}{l}\text { 7. } 97 \\
2.95 \\
3.60 \\
4.96 \\
4.94\end{array}$ & $\begin{array}{l}\text { 5. } 68 \\
\text { 3. } 49 \\
\text { 5. } 07 \\
\text { 3. } 59 \\
\text { 3. } 58\end{array}$ & $\begin{array}{l}\text { 5. } 22 \\
\text { 2. } 62 \\
\text { 3. } 40 \\
\text { 3. } 49 \\
\text { 3. } 51\end{array}$ \\
\hline $\begin{aligned} 10.27 \\
10.18 \\
10.16 \\
10.15 \\
9.85\end{aligned}$ & $\begin{array}{l}\text { "Ianthinite" } \\
\text { Tyuyamunite } \\
\text { Uranospinite. } \\
\text { Novacekite } \\
\text { Saléeite }\end{array}$ & $\begin{array}{l}\text { 5. } 18 \\
\text { 5. } 02 \\
\text { 3. } 56 \\
\text { 3. } 58 \\
\text { 3. } 49\end{array}$ & $\begin{array}{l}\text { 3. } 27 \\
3.20 \\
4.90 \\
\text { 5. } 06 \\
\text { 4. } 95\end{array}$ & $\begin{array}{l}\text { 4. } 06 \\
\text { 2. } 04 \\
\text { 3. } 39 \\
\text { 3. } 35 \\
\text { 2. } 19\end{array}$ \\
\hline $\begin{array}{l}9.82 \\
9.69 \\
9.14 \\
9.03 \\
8.93\end{array}$ & $\begin{array}{l}\text { Sengierite } \\
\text { Sabugalite } \\
\text { Abernathyite } \\
\text { Hydrogen autunite } \\
\text { Metazeunerite }\end{array}$ & $\begin{array}{l}\text { 4. } 91 \\
\text { 4. } 86 \\
\text { 3. } 84 \\
\text { 3. } 80 \\
\text { 3. } 72\end{array}$ & $\begin{array}{l}\text { 3. } 735 \\
\text { 3. } 47 \\
\text { 3. } 34 \\
\text { 3. } 27 \\
\text { 3. } 30\end{array}$ & $\begin{array}{l}\text { 3. } 197 \\
\text { 2. } 19 \\
\text { 3. } 59 \\
\text { 3. } 51 \\
\text { 3. } 56\end{array}$ \\
\hline $\begin{array}{l}\text { 8. } 85 \\
8.76 \\
8.76 \\
8.68 \\
8.66\end{array}$ & $\begin{array}{l}\text { Uranospinite. } \\
\text { Metazeunerite } \\
\text { Swartzite } \\
\text { Liebigite } \\
\text { Metatorbernite }\end{array}$ & $\begin{array}{l}\text { 3. } 59 \\
\text { 3. } 71 \\
\text { 5. } 50 \\
\text { 6. } 81 \\
\text { 3. } 69\end{array}$ & $\begin{array}{l}\text { 3. } 34 \\
\text { 3. } 28 \\
\text { 7. } 31 \\
\text { 5. } 40 \\
\text { 3. } 24\end{array}$ & $\begin{array}{l}\text { 5. } 10 \\
\text { 3. } 55 \\
\text { 4. } 82 \\
\text { 4. } 55 \\
\text { 3. } 51\end{array}$ \\
\hline $\begin{array}{l}\text { 8. } 59 \\
8.51 \\
8.51 \\
\text { 8. } 43 \\
8.42\end{array}$ & $\begin{array}{l}\text { Troegerite } \\
\text { Metatyuyamunite } \\
\text { Meta-autunite } \\
\text { Metatyuyamunite } \\
\text { Sklodowskite }\end{array}$ & $\begin{array}{l}\text { 3. } 79 \\
\text { 4. } 22 \\
\text { 3. } 50 \\
\text { 4. } 17 \\
\text { 4. } 19\end{array}$ & $\begin{array}{l}\text { 3. } 30 \\
\text { 3. } 25 \\
\text { 3. } 63 \\
\text { 3. } 28 \\
\text { 3. } 27\end{array}$ & $\begin{array}{l}\text { 4. } 35 \\
\text { 3. } 04 \\
\text { 3. } 24 \\
\text { 3. } 03 \\
\text { 3. } 52\end{array}$ \\
\hline $\begin{array}{l}8.24 \\
8.19 \\
8.18 \\
7.97 \\
7.97\end{array}$ & $\begin{array}{l}\text { Rabbittite } \\
\text { Meta-uranocircite } \\
\text { Cuprosklodowskite } \\
\text { Andersonite } \\
\text { Renardite }\end{array}$ & $\begin{array}{r}\text { 11. } 28 \\
\text { 3. } 58 \\
4.09 \\
13.0 \\
\text { 3. } 99\end{array}$ & $\begin{array}{l}\text { 4. } 37 \\
\text { 5. } 37 \\
\text { 2. } 97 \\
\text { 5. } 68 \\
\text { 5. } 83\end{array}$ & $\begin{array}{l}\text { 4. } 71 \\
\text { 2. } 08 \\
\text { 4. } 82 \\
\text { 5. } 22 \\
\text { 2. } 88\end{array}$ \\
\hline $\begin{array}{l}7.88 \\
7.83 \\
7.83 \\
7.73 \\
7.66\end{array}$ & $\begin{array}{l}\text { Uranophane } \\
\text { Phosphuranylite } \\
\text { Beta-uranophane } \\
\text { Johannite } \\
\text { Bayleyite }\end{array}$ & $\begin{array}{r}3.94 \\
3.97 \\
3.90 \\
6.16 \\
13.1\end{array}$ & $\begin{array}{l}\text { 2. } 99 \\
\text { 5. } 83 \\
\text { 3. } 51 \\
\text { 3. 41 } \\
\text { 3. } 83\end{array}$ & $\begin{array}{l}2.91 \\
2.86 \\
\text { 3. } 19 \\
3.87 \\
2.69\end{array}$ \\
\hline
\end{tabular}




\begin{tabular}{|c|c|c|c|c|}
\hline $\begin{array}{l}\text { Darkest line } \\
\text { (angstrom) }\end{array}$ & Mineral & \multicolumn{3}{|c|}{$\begin{array}{l}\text { Three next darkest } \\
\text { lines (angstrom) }\end{array}$} \\
\hline $\begin{array}{l}\text { 7. } 63 \\
7.58 \\
7.53 \\
\text { 7. } 50 \\
7.49\end{array}$ & $\begin{array}{l}\text { Epi-ianthinite... } \\
\text { Ianthinite } \\
\text { Billietite } \\
\text { Becquerelite. } \\
\text { Schoepite. }\end{array}$ & $\begin{array}{l}\text { 3. } 78 \\
\text { 3. } 78 \\
\text { 3. } 77 \\
\text { 3. } 22 \\
\text { 3. } 26\end{array}$ & $\begin{array}{l}\text { 3. } 20 \\
\text { 3. } 58 \\
\text { 3. } 17 \\
\text { 3. } 75 \\
\text { 3. } 64\end{array}$ & $\begin{array}{l}\text { 3. } 36 \\
\text { 3. } 34 \\
\text { 2. } 03 \\
\text { 3. } 56 \\
\text { 2. } 60\end{array}$ \\
\hline $\begin{array}{l}\text { 7. } 31 \\
\text { 7. } 31 \\
\text { 7. } 26 \\
\text { 7. } 19 \\
\text { 7. } 12\end{array}$ & 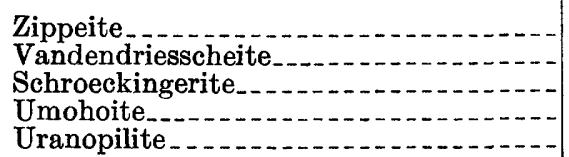 & $\begin{array}{l}\text { 3. } 66 \\
\text { 3. } 19 \\
\text { 4. } 796 \\
\text { 4. } 80 \\
9.18\end{array}$ & $\begin{array}{l}\text { 3. } 15 \\
\text { 3. } 58 \\
\text { 8. } 48 \\
\text { 3. } 60 \\
\text { 4. } 28\end{array}$ & $\begin{array}{l}\text { 3. } 51 \\
1.989 \\
2.876 \\
14.3 \\
3.65\end{array}$ \\
\hline $\begin{array}{l}\text { 7. } 10 \\
\text { 7. } 06 \\
6.81 \\
6.56 \\
6.28\end{array}$ & $\begin{array}{l}\text { Masuyite } \\
\text { Zippeite } \\
\text { Liebigite. } \\
\text { Carnotite. } \\
\text { Curite }\end{array}$ & $\begin{array}{l}\text { 3. } 15 \\
\text { 3. } 12 \\
\text { 8. } 68 \\
\text { 3. } 12 \\
\text { 3. } 97\end{array}$ & $\begin{array}{l}\text { 3. } 54 \\
\text { 3. } 51 \\
\text { 5. } 40 \\
\text { 3. } 53 \\
\text { 3. } 14\end{array}$ & $\begin{array}{l}1.984 \\
2.86 \\
4.55 \\
4.25 \\
3.53\end{array}$ \\
\hline $\begin{array}{l}\text { 6. } 16 \\
\text { 5. } 68 \\
\text { 5. } 50 \\
\text { 5. } 22 \\
\text { 5. } 02\end{array}$ & $\begin{array}{l}\text { Johannite } \\
\text { Andersonite } \\
\text { Swartzite } \\
\text { Andersonite } \\
\text { Tyuyamunite }\end{array}$ & $\begin{array}{l}\text { 7. } 73 \\
\text { 13. } 0 \\
8.76 \\
13.0 \\
10.18\end{array}$ & $\begin{array}{l}\text { 3. } 41 \\
\text { 7. } 97 \\
7.31 \\
\text { 7. } 97 \\
\text { 3. } 20\end{array}$ & $\begin{array}{l}\text { 3. } 87 \\
\text { 5. } 22 \\
4.82 \\
\text { 5. } 68 \\
\text { 2. } 04\end{array}$ \\
\hline $\begin{array}{l}\text { 4. } 94 \\
\text { 4. } 89 \\
\text { 4. } 86 \\
\text { 4. } 695 \\
\text { 4. } 69\end{array}$ & $\begin{array}{l}\text { Torbernite } \\
\text { Bassetite } \\
\text { Sabugalite } \\
\text { Thorogummite } \\
\text { Thorite }\end{array}$ & $\begin{array}{l}\text { 10. } 3 \\
\text { 3. } 46 \\
\text { 9. } 69 \\
\text { 3. } 537 \\
\text { 3. } 56\end{array}$ & $\begin{array}{l}\text { 3. } 58 \\
8.59 \\
3.47 \\
2.653 \\
1.84\end{array}$ & $\begin{array}{l}\text { 3. } 51 \\
2.20 \\
2.19 \\
1.818 \\
2.66\end{array}$ \\
\hline $\begin{array}{l}\text { 4. } 66 \\
\text { 4. } 60 \\
\text { 4. } 48 \\
\text { 4. } 44 \\
\text { 4. } 29\end{array}$ & $\begin{array}{l}\text { Coffinite } \\
\text { Rutherfordine } \\
\text { Soddyite } \\
\text { Vandenbrandeite } \\
\text { Dumontite }\end{array}$ & $\begin{array}{l}\text { 3. } 47 \\
\text { 3. } 21 \\
\text { 3. } 32 \\
\text { 5. } 26 \\
\text { 3. } 02\end{array}$ & $\begin{array}{l}\text { 2. } 64 \\
\text { 4. } 29 \\
\text { 6. } 14 \\
\text { 2. } 97 \\
\text { 3. } 75\end{array}$ & $\begin{array}{l}\text { 1. } 801 \\
\text { 3. } 90 \\
\text { 2. } 69 \\
2.59 \\
\text { 3. } 49\end{array}$ \\
\hline $\begin{array}{l}\text { 4. } 28 \\
\text { 4. } 25 \\
\text { 4. } 09 \\
\text { 3. } 99 \\
\text { 3. } 97\end{array}$ & $\begin{array}{l}\text { Vandenbrandeite } \\
\text { Parsonsite } \\
\text { Cuprosklodowskite } \\
\text { Renardite } \\
\text { Curite }\end{array}$ & $\begin{array}{l}\text { 2. } 91 \\
3.28 \\
8.18 \\
7.97 \\
6.28\end{array}$ & $\begin{array}{l}\text { 5. } 05 \\
2.132 \\
2.97 \\
5.83 \\
\text { 3. } 14\end{array}$ & $\begin{array}{l}2.55 \\
\text { 1. } 661 \\
4.82 \\
2.88 \\
\text { 3. } 53\end{array}$ \\
\hline $\begin{array}{l}\text { 3. } 94 \\
\text { 3. } 90 \\
\text { 3. } 78 \\
\text { 3. } 77 \\
\text { 3. } 71\end{array}$ & $\begin{array}{l}\text { Uranophane } \\
\text { Beta-uranophane } \\
\text { Epi-ianthinite } \\
\text { Billietite } \\
\text { Metazeunerite }\end{array}$ & $\begin{array}{l}\text { 7. } 88 \\
7.83 \\
7.63 \\
7.53 \\
8.76\end{array}$ & $\begin{array}{l}\text { 2. } 99 \\
\text { 3. } 51 \\
\text { 3. } 20 \\
\text { 3. } 17 \\
\text { 3. } 28\end{array}$ & $\begin{array}{l}\text { 2. } 91 \\
\text { 3. } 19 \\
\text { 3. } 36 \\
\text { 2. } 03 \\
\text { 3. } 55\end{array}$ \\
\hline $\begin{array}{l}\text { 3. } 69 \\
\text { 3. } 60 \\
\text { 3. } 59 \\
\text { 3. } 58 \\
\text { 3. } 56\end{array}$ & $\begin{array}{l}\text { Metatorbernite } \\
\text { Zeunerite } \\
\text { Uranospinite } \\
\text { Meta-uranocircite } \\
\text { Uranospinite }\end{array}$ & $\begin{array}{r}8.66 \\
10.33 \\
8.85 \\
8.19 \\
10.16\end{array}$ & $\begin{array}{l}\text { 3. } 24 \\
\text { 5. } 07 \\
\text { 3. } 34 \\
\text { 2. } 08 \\
\text { 3. } 39\end{array}$ & $\begin{array}{l}\text { 3. } 51 \\
\text { 3. } 40 \\
\text { 5. } 10 \\
\text { 5. } 37 \\
\text { 4. } 90\end{array}$ \\
\hline $\begin{array}{l}\text { 3. } 56 \\
\text { 3. } 537 \\
3.50 \\
\text { 3. } 49 \\
\text { 3. } 47\end{array}$ & $\begin{array}{l}\text { Thorite } \\
\text { Thorogummite } \\
\text { Meta-autunite } \\
\text { Saléeite } \\
\text { Coffinite. }\end{array}$ & $\begin{array}{l}\text { 1. } 84 \\
\text { 4. } 695 \\
8.51 \\
9.85 \\
\text { 4. } 66\end{array}$ & $\begin{array}{l}\text { 4. } 69 \\
\text { 2. } 653 \\
\text { 3. } 63 \\
\text { 4. } 95 \\
\text { 2. } 64\end{array}$ & $\begin{array}{l}\text { 2. } 66 \\
\text { 1. } 818 \\
\text { 3. } 24 \\
\text { 2. } 19 \\
\text { 1. } 801\end{array}$ \\
\hline
\end{tabular}




\begin{tabular}{|c|c|c|c|c|}
\hline $\begin{array}{l}\text { Darkest line } \\
\text { (angstrom) }\end{array}$ & Mineral & \multicolumn{3}{|c|}{$\begin{array}{c}\text { Three next darkest } \\
\text { lines (angstrom) }\end{array}$} \\
\hline $\begin{array}{l}\text { 3. } 46 \\
\text { 3. } 45 \\
\text { 3. } 43 \\
\text { 3. } 42 \\
\text { 3. } 41\end{array}$ & $\begin{array}{l}\text { Bassetite } \\
\text { Fourmarierite } \\
\text { Fourmarierite.... } \\
\text { Brannerite } \\
\text { Brannerite }\end{array}$ & $\begin{array}{l}\text { 4. } 89 \\
\text { 3. } 09 \\
\text { 3. } 08 \\
\text { 1. } 903 \\
\text { 1. } 903\end{array}$ & $\begin{array}{l}\text { 8. } 59 \\
\text { 1. } 907 \\
\text { 1. } 903 \\
\text { 2. } 455 \\
\text { 2. } 462\end{array}$ & $\begin{array}{l}\text { 2. } 20 \\
\text { 1. } 729 \\
\text { 1. } 990 \\
\text { 2. } 276 \\
\text { 2. } 276\end{array}$ \\
\hline $\begin{array}{l}\text { 3. } 41 \\
\text { 3. } 34 \\
\text { 3. } 32 \\
\text { 3. } 26 \\
\text { 3. } 26\end{array}$ & 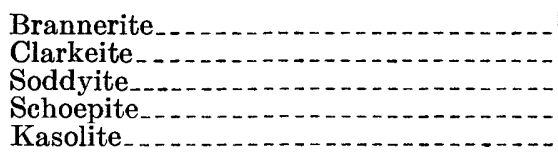 & $\begin{array}{l}\text { 1. } 903 \\
\text { 3. } 17 \\
\text { 4. } 48 \\
\text { 7. } 49 \\
\text { 2. } 93\end{array}$ & $\begin{array}{l}\text { 2. } 449 \\
\text { 5. } 77 \\
\text { 6. } 14 \\
\text { 3. } 64 \\
\text { 4. } 19\end{array}$ & $\begin{array}{l}\text { 2. } 270 \\
\text { 1. } 968 \\
2.69 \\
\text { 2. } 60 \\
\text { 3. } 53\end{array}$ \\
\hline $\begin{array}{l}\text { 3. } 22 \\
\text { 3. } 21 \\
\text { 3. } 21 \\
\text { 3. } 20 \\
\text { 3. } 17\end{array}$ & $\begin{array}{l}\text { Vandendriesscheite } \\
\text { Rutherfordine } \\
\text { Thorianite } \\
\text { Samiresite } \\
\text { Clarkeite }\end{array}$ & $\begin{array}{l}\text { 3. } 61 \\
\text { 4. } 60 \\
\text { 1. } 68 \\
\text { 2. } 50 \\
\text { 3. } 34\end{array}$ & $\begin{array}{l}\text { 7. } 41 \\
4.29 \\
\text { 1. } 97 \\
\text { 4. } 05 \\
\text { 5. } 77\end{array}$ & $\begin{array}{l}\text { 2. } 55 \\
\text { 3. } 90 \\
\text { 2. } 78 \\
\text { 1. } 83 \\
\text { 1. } 968\end{array}$ \\
\hline $\begin{array}{l}\text { 3. } 16 \\
\text { 3. } 14 \\
\text { 3. } 12 \\
\text { 3. } 11 \\
\text { 3. } 11\end{array}$ & $\begin{array}{l}\text { Uranospherite } \\
\text { Uraninite } \\
\text { Delorenzite } \\
\text { Walpurgite } \\
\text { Cerianite }\end{array}$ & $\begin{array}{l}3.87 \\
1.645 \\
2.87 \\
3.05 \\
1.90\end{array}$ & $\begin{array}{l}\text { 3. } 47 \\
\text { 1. } 926 \\
\text { 2. } 67 \\
\text { 3. } 25 \\
\text { 1. } 62\end{array}$ & $\begin{array}{l}\text { 5. } 25 \\
\text { 1. } 051 \\
2.52 \\
9.9 \\
\text { 2. } 69\end{array}$ \\
\hline $\begin{array}{l}\text { 3. } 09 \\
\text { 3. } 09 \\
\text { 3. } 08 \\
\text { 3. } 07 \\
\text { 3. } 02\end{array}$ & $\begin{array}{l}\text { Fourmarierite } \\
\text { Huttonite } \\
\text { Fourmarierite } \\
\text { Cheralite } \\
\text { Dumontite }\end{array}$ & $\begin{array}{l}\text { 3. } 45 \\
\text { 2. } 89 \\
\text { 3. } 43 \\
\text { 3. } 26 \\
\text { 4. } 29\end{array}$ & $\begin{array}{l}\text { 1. } 907 \\
\text { 4. } 23 \\
\text { 1. } 903 \\
\text { 2. } 86 \\
\text { 3. } 75\end{array}$ & $\begin{array}{l}\text { 1. } 729 \\
\text { 3. } 29 \\
\text { 1. } 990 \\
\text { 4. } 14 \\
\text { 3. } 49\end{array}$ \\
\hline $\begin{array}{l}\text { 2. } 98 \\
2.98 \\
2.96 \\
\text { 2. } 95 \\
\text { 2. } 94\end{array}$ & $\begin{array}{l}\text { Betafite } \\
\text { Microlite } \\
\text { Uvanite } \\
\text { Uvanite } \\
\text { Uvanite }\end{array}$ & $\begin{array}{l}\text { 1. } 82 \\
\text { 1. } 836 \\
\text { 1. } 71 \\
\text { 2. } 22 \\
\text { 1. } 71\end{array}$ & $\begin{array}{l}\text { 1. } 55 \\
\text { 1. } 563 \\
\text { 5. } 9 \\
\text { 1. } 70 \\
\text { 2. } 24\end{array}$ & $\begin{array}{l}\text { 3. } 99 \\
\text { 5. } 98 \\
\text { 2. } 24 \\
\text { 1. } 47 \\
\text { 1. } 484\end{array}$ \\
\hline $\begin{array}{l}\text { 2. } 94 \\
2.93 \\
2.93 \\
2.89 \\
\text { 2. } 87\end{array}$ & 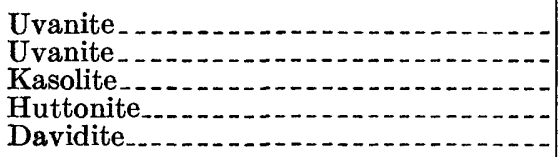 & $\begin{array}{l}\text { 4. } 60 \\
\text { 2. } 20 \\
\text { 3. } 26 \\
\text { 3. } 09 \\
\text { 1. } 70\end{array}$ & $\begin{array}{l}\text { 5. } 79 \\
9.56 \\
\text { 4. } 19 \\
\text { 4. } 23 \\
\text { 3. } 40\end{array}$ & $\begin{array}{l}1.71 \\
1.70 \\
3.53 \\
3.29 \\
3.23\end{array}$ \\
\hline $\begin{array}{l}\text { 2. } 86 \\
\text { 1. } 84 \\
\text { 1. } 70 \\
\text { 1. } 69 \\
\text { 1. } 68 \\
\text { 1. } 645\end{array}$ & 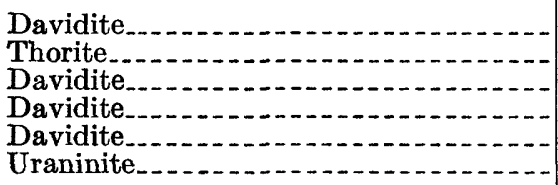 & $\begin{array}{l}\text { 1. } 60 \\
\text { 3. } 56 \\
\text { 1. } 69 \\
\text { 1. } 70 \\
\text { 3. } 22 \\
\text { 3. } 14\end{array}$ & $\begin{array}{l}\text { 1. } 44 \\
\text { 4. } 69 \\
\text { 1. } 435 \\
\text { 1. } 435 \\
\text { 2. } 48 \\
\text { 1. } 926\end{array}$ & $\begin{array}{l}\text { 1. } 81 \\
\text { 2. } 66 \\
1.59 \\
1.59 \\
1.04 \\
1.051\end{array}$ \\
\hline
\end{tabular}




\section{MINERALS}

The following tables give the complete list of $d$-spacings for each of the known uranium and thorium minerals for which data are available. The minerals are listed alphabetically.

The locality from which the X-rayed sample was obtained is cited, and a reference given where the data were not obtained from the Harvard or Geological Survey standard files.

ABERNATHYITE

$\mathrm{K}_{2}\left(\mathrm{UO}_{2}\right)_{2}\left(\mathrm{AsO}_{4}\right)_{2} \cdot 8 \mathrm{H}_{2} \mathrm{O}$

Data from Thompson, Ingram, and Gross (1956) on material from the Fuemrol No. 2 mine, Temple Mountain, Emery County, Utah.

\begin{tabular}{|c|c|c|c|c|}
\hline$d(\mathrm{~A})$ & $I$ & $\mathbf{h k l}$ & $d(\mathrm{~A})$ & $I$ \\
\hline 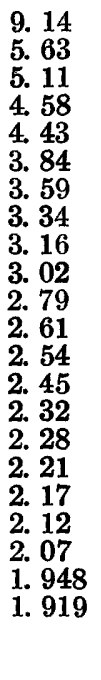 & $\begin{array}{l}10^{\mathrm{b}} \\
7 \\
4 \\
4 \\
4 \\
8^{\mathrm{b}} \\
7 \\
8 \\
1 \\
5 \\
\mathbf{6}^{\mathrm{b}} \\
\mathbf{5}^{\mathrm{b}} \\
\mathbf{3} \\
5 \\
3 \\
6 \\
6 \\
2 \\
6 \\
6 \\
3 \\
1\end{array}$ & $\begin{array}{l}001 \\
101 \\
110 \\
002 \\
111 \\
102 \\
200 \\
112,201 \\
003,211 \\
202,103 \\
113,212 \\
220 \\
221 \\
203,301 \\
310,004 \\
222,213,311 \\
104 \\
302 \\
114\end{array}$ & $\begin{array}{l}1.879 \\
1.855 \\
1.822 \\
1.797 \\
1.780 \\
1.712 \\
1.667 \\
1.625 \\
1.606 \\
1.582 \\
1.514 \\
1.482 \\
1.458 \\
1.421 \\
1.372 \\
1.359 \\
1.300 \\
1.280 \\
1.261 \\
1.240 \\
1.212 \\
1.206 \\
1.198 \\
1.187\end{array}$ & $\begin{array}{l}3 \\
1 \\
5 \\
3 \\
3 \\
6 \\
3 \\
3 \\
6 \\
3 \\
3 \\
3 \\
3 \\
6 \\
3 \\
1 \\
1 \\
5 \\
4 \\
1 \\
1 b \\
1 b \\
3 \\
3 \\
1\end{array}$ \\
\hline
\end{tabular}

broad. 


\section{ABSITE}

A thorian variety of brannerite.

$$
\begin{gathered}
\text { ANDERSONITE } \\
\mathrm{Na}_{2} \mathrm{Ca}\left(\mathrm{UO}_{2}\right)\left(\mathrm{CO}_{3}\right)_{3} \cdot 6 \mathrm{H}_{2} \mathrm{O}
\end{gathered}
$$

Data from Axelrod, Grimaldi, Milton, and Murata (1951) on material from the Hillside mine, Yavapai County, Ariz.

\begin{tabular}{c|r||l|l}
\hline$d(\mathrm{~A})$ & $I$ & $d(\mathrm{~A})$ & $I$ \\
\hline 13.0 & 10 & 2.21 & 7 \\
9.51 & 3 & 2.04 & 4 \\
7.97 & 10 & 2.01 & 4 \\
6.56 & 2 & 1.977 & 4 \\
5.68 & 10 & 1.957 & 4 \\
5.22 & 10 & 1.933 & 4 \\
4.35 & 6 & 1.895 & 4 \\
4.19 & 6 & 1.852 & 6 \\
4.04 & 4 & 1.749 & 5 \\
3.82 & 3 & 1.684 & 3 \\
3.71 & 8 & 1.573 & 4 \\
3.49 & 2 & 1.393 & 3 \\
3.34 & 2 & 1.344 & 3 \\
3.15 & 5 & 1.262 & 3 \\
3.00 & 7 & 1.232 & 3 \\
2.79 & 6 & 1.038 & 2 \\
2.45 & 5 & .980 & 2 \\
2.39 & 5 & .951 & 2 \\
2.36 & 4 & .819 & 2 \\
& & .801 & 2 \\
\hline
\end{tabular}




\section{AUTUNITE}

$$
\mathrm{Ca}\left(\mathrm{UO}_{2}\right)_{2}\left(\mathrm{PO}_{4}\right)_{2} \cdot 8-12 \mathrm{H}_{2} \mathrm{O}
$$

Fully hydrated synthetic material. See further under meta-autunite.

\begin{tabular}{l|l||l|l}
\hline$d(\mathrm{~A})$ & $I$ & $d(\mathrm{~A})$ & $I$ \\
\hline 10.33 & 10 & 1.539 & \\
8.97 & 1 & 1.506 & 2 \\
6.70 & 2 & 1.473 & 1 \\
4.96 & 8 & 1.412 & 1 \\
4.48 & 6 & 1.357 & 4 \\
3.59 & 7 & 1.323 & 2 \\
3.49 & 7 & 1.288 & 1 \\
3.33 & 7 & 1.261 & 2 \\
3.12 & 1 & 1.223 & 2 \\
2.88 & 5 & 1.191 & 3 \\
2.71 & 2 & 1.159 & 1 \\
2.49 & 2 & 1.133 & 1 \\
2.40 & 2 & 1.101 & 1 \\
2.21 & 3 & 1.072 & 2 \\
2.15 & 3 & 1.036 & 1 \\
2.03 & 2 & 1.027 & 1 \\
1.911 & 1 & .985 & 3 \\
1.871 & $1 \mathrm{~d}$ & .971 & 1 \\
1.823 & 1 & .966 & 1 \\
1.750 & 2 & .949 & 1 \\
1.720 & 1 & .935 & 1 \\
1.644 & 1 & .930 & 1 \\
1.617 & 4 & .916 & 2 \\
1.566 & 2 & .905 & 1 \\
\hline
\end{tabular}

d Diffuse.

\section{BASSETITE}

$$
\mathrm{Fe}\left(\mathrm{UO}_{2}\right)_{2}\left(\mathrm{PO}_{4}\right)_{2} \cdot 8 \mathrm{H}_{2} \mathrm{O}
$$

Data from Frondel (1954) on material from Cornwall, England.

\begin{tabular}{l|r||l|c}
\hline$d(\mathrm{~A})$ & $I$ & $d(\mathrm{~A})$ & $I$ \\
\hline 8.59 & & & \\
4.89 & 10 & 2.13 & 1 \\
4.66 & $1 / 2$ & 1.07 & $1 / 2$ \\
4.24 & 3 & 1.921 & $1 / 2 \mathrm{~d}$ \\
4.05 & 3 & 1.830 & 2 \\
3.46 & 10 & 1.783 & $1 / 2$ \\
3.32 & $1 / 2$ & 1.708 & 2 \\
3.10 & 1 & $1 / 2 \mathrm{~d}$ \\
2.96 & 3 & 1.627 & 1 vd \\
2.85 & 2 & 1.457 & 1 vd \\
2.72 & $1 / 2$ & $1 / 2$ vd \\
2.55 & $1 / 2 \mathrm{~d}$ & 1.374 & $1 / 2$ vd \\
2.46 & $2 \mathrm{~d}$ & 1.191 & $1 / 2$ vd \\
2.34 & $1 / 2$ & 1.162 & 1 vd \\
2.26 & $1 / 2$ & 1.128 & $1 / 2$ vd \\
2.20 & 6 & 1.106 & $1 / 2$ vd \\
\hline
\end{tabular}

d Diffuse. vi Very diffuse. 
BAYLEYITE

$$
\mathrm{Mg}_{2}\left(\mathrm{UO}_{2}\right)\left(\mathrm{CO}_{3}\right)_{3} \cdot 18 \mathrm{H}_{2} \mathrm{O}
$$

Data from Axelrod, Grimaldi, Milton, and Murata (1951) on material from the Hillside mine, Yavapai County, Ariz. Bayleyite dehydrates readily, and data also are given here for this phase.

\begin{tabular}{|c|c|c|c|c|}
\hline \multicolumn{3}{|c|}{ Bayleyite, Arizona } & \multicolumn{2}{|c|}{$\begin{array}{c}\text { Dehydration product } \\
\text { of bayleyite, } \\
\text { Arizona }\end{array}$} \\
\hline$d(\mathrm{~A})$ & $I$ & hkl & $d(\mathrm{~A})$ & $I$ \\
\hline $\begin{array}{l}13.1 \\
7.66 \\
6.53 \\
5.85 \\
5.08 \\
4.89 \\
4.71 \\
\text { 4. } 54 \\
\text { 4. } 41 \\
\text { 3. } 83 \\
3.70 \\
3.26 \\
3.15 \\
3.05 \\
2.96 \\
2.88 \\
2.69 \\
2.42 \\
2.37 \\
2.30 \\
2.21 \\
2.12 \\
1.908 \\
1.796 \\
1.754 \\
1.682 \\
1.378 \\
1.247\end{array}$ & $\begin{array}{c}9 \\
10 \\
4 \\
4 b^{b} \\
4 \\
4 \\
1 \\
2 \\
2 \\
6 \\
2 \\
4 \\
2 \\
2 \\
2 \\
4 \\
5 \\
2 \\
2 \\
2 \\
5 \\
4 \\
3 \\
2 b \\
2 b \\
2 b \\
1 \\
1 \\
1\end{array}$ & $\begin{array}{l}200,110 \\
310,020 \\
400,220,001 \\
201,111,11 \overline{1} \\
311 \\
021,31 \overline{1}, 401 \\
221,22 \overline{1} \\
40 \overline{1} \\
330,600 \\
620,040 \\
331,710,530,240 \\
002 \\
11 \overline{2}, 212 \\
312,730,911\end{array}$ & $\begin{array}{l}\text { 8. } 27 \\
7.25 \\
6.24 \\
5.54 \\
5.34 \\
5.13 \\
4.77 \\
\text { 4. } 62 \\
\text { 4. 51 } \\
\text { 4. 42 } \\
\text { 4. 23 } \\
4.00 \\
3.75 \\
3.56 \\
3.49 \\
3.20 \\
3.11 \\
3.07 \\
3.02 \\
2.89 \\
2.86 \\
2.76 \\
2.71 \\
2.64 \\
2.47 \\
2.44 \\
2.32 \\
2.26 \\
2.14 \\
2.12 \\
2.08 \\
2.05 \\
1.973 \\
1.881 \\
1.848 \\
1.811 \\
1.787 \\
1.752 \\
1.695 \\
1.578 \\
1.551 \\
1.352\end{array}$ & 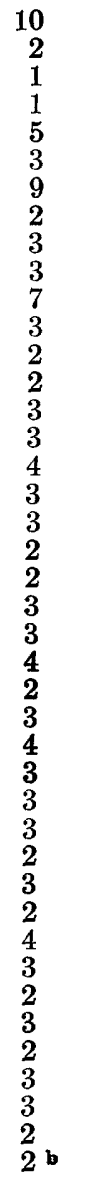 \\
\hline
\end{tabular}

b Broad. 
$7 \mathrm{UO}_{3} \cdot \mathrm{H}_{2} \mathrm{O}$

Data from J. W. Frondel and Cuttitta (1953) for material from the Katanga district, Belgian Congo. A virtually identical pattern is given by synthetic material.

\begin{tabular}{|c|c|c|c|}
\hline$d(\mathrm{~A})$ & $I$ & $d(\mathrm{~A})$ & $I$ \\
\hline 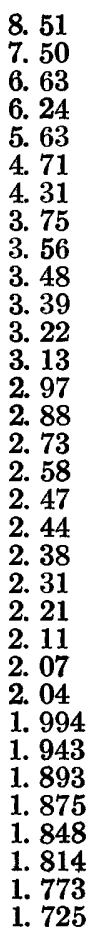 & $\begin{array}{r}1 \\
10 \\
2 \\
1 \\
1 \\
6 \\
1 \\
8 \\
8 \\
2 \\
2 \\
9 \\
1 \\
2 \\
3 \\
1 \\
7 \\
2 \\
2 \\
2 \\
3 \\
1 \\
2 \\
3 \\
4 \\
4 \\
5 \\
2 \\
2 \\
2 \\
1 \\
3 \\
3\end{array}$ & $\begin{array}{l}1.681 \\
1.614 \\
1.600 \\
1.564 \\
1.541 \\
1.451 \\
1.411 \\
1.378 \\
1.332 \\
1.289 \\
1.271 \\
1.240 \\
1.224 \\
1.207 \\
1.188 \\
1.169 \\
1.152 \\
1.134 \\
1.121 \\
1.108 \\
1.054 \\
1.029 \\
.987 \\
.967 \\
.955 \\
.944 \\
.933 \\
.914 \\
.887 \\
.859 \\
.842 \\
.836 \\
.834\end{array}$ & $\begin{array}{l}2 \\
2 \\
2 \\
2 \\
1 \\
2 \\
3 \\
3 \\
1 \\
1 \\
1 \\
1 \\
1 \\
1 \\
1 \\
1 \\
1 \\
1 \\
1 \\
1 \\
1 \\
1 \\
1 \\
1 \\
1 \\
1 \\
1 \\
1 \\
1 \\
1 \\
1 \\
1 \\
1\end{array}$ \\
\hline
\end{tabular}




$$
\text { (U, Ca) (Nb, Ta, Ti) }{ }_{3} \mathrm{O}_{8} \cdot n \mathrm{H}_{2} \mathrm{O}
$$

The chemical composition of betafite varies considerably but detailed information is lacking about the attendant variation in spacings. Samiresite, apparently a variety of betafite high in lead, may be a distinct species; powder data for this mineral are given below. Betafite and its compositional variants apparently always are metamict. The present data are for authentic but unanalyzed material heated in air to $1000^{\circ} \mathrm{C}$.

\begin{tabular}{|c|c|c|c|}
\hline \multicolumn{2}{|c|}{$\begin{array}{l}\text { Betafite, Antanifotsy, } \\
\text { Madagascar }\end{array}$} & \multicolumn{2}{|c|}{ Samiresite, Madagascar } \\
\hline$d(\mathrm{~A})$ & $I$ & $d(\mathrm{~A})$ & $I$ \\
\hline $\begin{array}{l}3.99 \\
\text { 3. } 55 \\
\text { 3. } 28 \\
3.19 \\
2.98 \\
\text { 2. } 57 \\
2.49 \\
2.20 \\
2.00 \\
1.82 \\
1.70 \\
1.63 \\
1.59 \\
1.55 \\
1.485 \\
1.355 \\
1.290 \\
1.181 \\
1.154 \\
1.050 \\
1.025 \\
.990\end{array}$ & $\begin{array}{r}3 \\
1 \\
1 \\
2 \\
10 \\
2 \\
2 \\
3 \\
1 \\
1 \\
8 \\
2 \\
1 \\
1 \\
6 \\
1 \\
1 \\
1 \\
2 \\
3 \\
1 \\
1 \\
2\end{array}$ & $\begin{array}{l}4.05 \\
3.55 \\
3.20 \\
2.99 \\
2.81 \\
2.61 \\
2.50 \\
2.02 \\
1.83 \\
1.71 \\
1.67 \\
1.59 \\
1.48 \\
1.36 \\
1.25 \\
1.20 \\
1.153\end{array}$ & $\begin{array}{r}6 \\
2 \\
2 \\
10 \\
2 \\
1 \\
1 \\
8 \\
2 \\
2 \\
5 \\
3 \\
3 \\
1 \\
2 \\
2 \\
1 \\
1 \\
2\end{array}$ \\
\hline
\end{tabular}


BETA-URANOPHANE

$$
\mathrm{Ca}\left(\mathrm{UO}_{2}\right)_{2}\left(\mathrm{SiO}_{3}\right)_{2}(\mathrm{OH})_{2} \cdot 5 \mathrm{H}_{2} \mathrm{O}
$$

Material from Joachimsthal, Bohemia. Direct comparison of films of material from numerous localities shows no significant variations in spacing. Beta-uranophane is said to be converted to its polymorph uranophane on heavy grinding. Care also must be taken to avoid preferred orientation in the preparation of the mount. Uranophane, beta-uranophane, and some types of kasolite are similar in the hand specimen, but their X-ray patterns are distinctive.

\begin{tabular}{|c|c|c|c|c|c|}
\hline$d(\mathrm{~A})$ & $I$ & $d(\mathrm{~A})$ & $I$ & $d(\mathrm{~A})$ & $I$ \\
\hline $\begin{array}{l}7.83 \\
6.66 \\
6.15 \\
5.07 \\
4.85 \\
4.55 \\
\text { 4. } 11 \\
\text { 3. } 90 \\
\text { 3. } 75 \\
\text { 3. } 51 \\
\text { 3. } 41 \\
3.35 \\
\text { 3. } 19 \\
3.04 \\
2.99 \\
2.91 \\
2.83 \\
2.78 \\
2.59\end{array}$ & $\begin{array}{r}10 \\
4 \\
4 \\
4 \\
4 \\
4 \\
3 \\
9 \\
1 \\
6 \\
1 \\
2 \\
5 \\
4 \\
3 \\
2 \\
4 \\
3 \\
5\end{array}$ & $\begin{array}{l}2.41 \\
2.36 \\
2.29 \\
2.21 \\
2.20 \\
2.17 \\
2.13 \\
2.11 \\
2.08 \\
2.04 \\
2.01 \\
1.98 \\
1.93 \\
1.877 \\
1.841 \\
1.807 \\
1.778 \\
1.734 \\
1.701\end{array}$ & $\begin{array}{l}2 \mathrm{2b} \\
2{ }^{\mathrm{b}} \\
1 \\
2 \\
2 \mathrm{~b} \\
2 \mathrm{~b} \\
1 \\
1 \\
1 \\
2 \\
1 \\
1 \\
1 \\
3 \\
3 \\
3 \\
3 \\
3 \\
3 \\
2 \\
3 \\
3 \\
2 \\
2 \mathrm{~b}\end{array}$ & $\begin{array}{l}1.658 \\
1.623 \\
1.590 \\
1.551 \\
1.528 \\
1.506 \\
1.484 \\
1.461 \\
1.441 \\
1.417 \\
1.388 \\
1.358 \\
1.310 \\
1.293 \\
1.274 \\
1.264 \\
1.186 \\
1.161\end{array}$ & $\begin{array}{l}1 \mathrm{~b} \\
3 \mathrm{~b} \\
1 / 2 \\
1 \\
1 \\
1 \\
1 \\
1 \\
1 \\
1 \\
1 \\
2 \mathrm{~b} \\
2 \mathrm{~b} \\
1 \mathrm{~b} \\
1 \mathrm{~b} \\
2 \\
2 \\
1 \mathrm{~b} \\
2 \mathrm{~b} \\
\mathrm{~b}\end{array}$ \\
\hline
\end{tabular}

b Broad. 


\section{BILLIETITE}

\section{$\mathrm{BaO} \cdot 6 \mathrm{UO}_{3} \cdot 11 \mathrm{H}_{2} \mathrm{O}$}

Material from Shinkolobwe, Katanga, Belgian Congo. See also J. W. Frondel and Cuttitta (1953). The pattern resembles that of becquerelite, with which mineral billietite is isostructural.

\begin{tabular}{|c|c|c|c|}
\hline$d(\mathrm{~A})$ & $I$ & $d(\mathrm{~A})$ & I \\
\hline $\begin{array}{l}7.53 \\
\text { 4. 59 } \\
\text { 3. } 77 \\
\text { 3. } 54 \\
\text { 3. } 17 \\
\text { 3. } 02 \\
\text { 2. } 89 \\
\text { 2. } 79 \\
\text { 2. } 56 \\
\text { 2. } 49 \\
\text { 2. } 30 \\
\text { 2. } 19 \\
\text { 2. } 10 \\
\text { 2. } 03 \\
1.970 \\
1.940 \\
1.898 \\
1.872 \\
1.812 \\
1.790 \\
1.676 \\
1.654 \\
1.604 \\
1.568 \\
1.530 \\
1.501 \\
1.459 \\
1.429 \\
1.381 \\
1.357 \\
1.310 \\
1.281\end{array}$ & $\begin{array}{c}10 \\
2 \\
9 \\
5{ }^{b} \\
8 \\
1 \\
1 \\
2 \\
3 \\
4 \\
2 \\
1{ }^{d} \\
3 \\
6 \\
4 \\
2 \\
1 \\
3 \\
1 \\
2 \\
3 \\
4 \\
1 \\
2 \\
1 d \\
2 \\
2 \\
1 \\
2 \\
1 \\
2 \\
2\end{array}$ & $\begin{array}{l}1.251 \\
1.226 \\
1.212 \\
1.189 \\
1.163 \\
1.140 \\
1.123 \\
1.097 \\
1.073 \\
1.058 \\
1.042 \\
1.026 \\
1.013 \\
1.003 \\
.987 \\
.981 \\
.944 \\
.931 \\
.914 \\
.898 \\
.884 \\
.871 \\
.858 \\
.852 \\
.843 \\
.831 \\
.824 \\
.783 \\
.783 \\
.776 \\
.774\end{array}$ & $\begin{array}{l}1 \\
1 \\
1 \\
1 \\
1 d \\
1 \\
2 \\
1 \\
1 \\
1 \\
2 \\
1 \\
1 \\
1 \\
1 \\
1 \\
1 \\
1 \\
1 \\
3 \\
1 \\
1 \\
1 \\
2 \\
2 \\
1 \\
1 \\
2 \\
2 \\
1 \\
4 \\
2 \\
2 \\
1\end{array}$ \\
\hline
\end{tabular}

b Broad. d Diffuse. vd Very diffuse. 


\section{BRANNERITE}

$$
\text { (U, Ca, Fe, } \mathrm{Th}, \mathrm{Y}_{3} \mathrm{Ti}_{5} \mathrm{O}_{16}(?)
$$

Brannerite apparently is always metamict. The data here given, from Pabst (1954), were obtained on material crystallized by heating in air to about $1000^{\circ} \mathrm{C}$. Minor variations in the spacings may be expected in material from other localities due to variation in composition. Further, the experimental conditions under which the recrystallization is effected may influence the results obtained.

\begin{tabular}{|c|c|c|c|c|c|}
\hline \multicolumn{2}{|c|}{ Mono County, Calif. } & \multicolumn{2}{|c|}{$\begin{array}{l}\text { Custer County, Idaho } \\
\text { (USNM 105793) }\end{array}$} & \multicolumn{2}{|c|}{$\begin{array}{l}\text { Bou-Azzer mine, } \\
\text { Morocco }\end{array}$} \\
\hline$d(\mathrm{~A})$ & $I$ & $d(\mathrm{~A})$ & $I$ & $d(\mathrm{~A})$ & $I$ \\
\hline 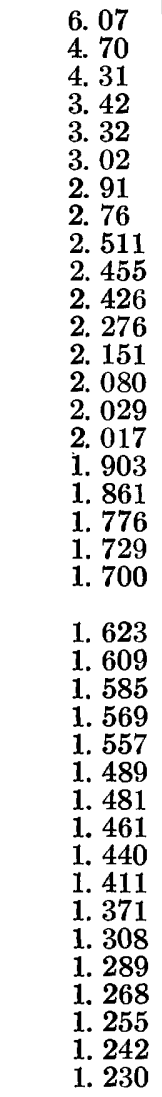 & 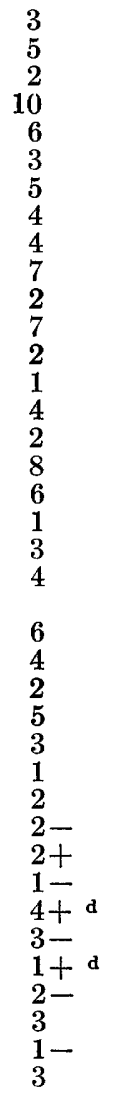 & $\begin{array}{l}\text { 6. } 03 \\
\text { 4. } 73 \\
\text { 4. } 32 \\
\text { 3. } 41 \\
\text { 3. } 32 \\
\text { 3. } 02 \\
2.92 \\
2.75 \\
2.508 \\
\text { 2. } 462 \\
2.432 \\
2.276 \\
2.161 \\
2.078 \\
2.032 \\
2.014 \\
1.903 \\
1.864 \\
1.778 \\
1.735 \\
1.702 \\
\text { 1. } 625 \\
1.609 \\
1.588 \\
1.570 \\
1.557 \\
\text { 1. } 484 \\
1.458 \\
1.444 \\
1.413 \\
1.370 \\
1.311 \\
1.287 \\
1.267 \\
1.253 \\
1.229\end{array}$ & $\begin{array}{l}4 \\
6 \\
3 \\
10 \\
6 \\
3+ \\
5 \\
4 \\
4 \\
6 \\
1 \\
6 \\
2 \\
1+ \\
4 \\
2 \\
8 \\
6 \\
1- \\
3 \\
3 \\
6 \\
2 \\
1- \\
5 \\
2 \\
2 d \\
2 \\
3 \\
1+ \\
5 d \\
2 \\
2 d \\
1 \\
3 \\
3- \\
3-\end{array}$ & 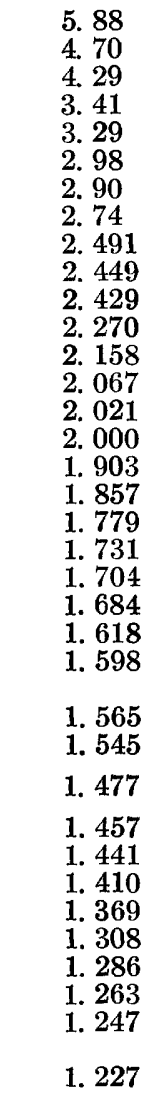 & $\begin{array}{l}3 \\
5 \\
2 \\
10 \\
6 \\
3 \\
5 \\
4 \\
4 \\
7 \\
2- \\
6 \\
2 \\
1 \\
4 \\
1- \\
7 \\
5 \\
1- \\
3 \\
2 \\
2 \\
7 \\
2 \\
? \\
6 \\
2 \\
3 \text { d } \\
2- \\
2 \\
2- \\
4 \\
3 \\
2 \\
1+ \\
3 \\
4 \\
4\end{array}$ \\
\hline
\end{tabular}

Diffuse. 


\section{CARNOTITE}

$$
\mathrm{K}_{2}\left(\mathrm{UO}_{2}\right)_{2}\left(\mathrm{VO}_{4}\right)_{2} \cdot 1-3 \mathrm{H}_{2} \mathrm{O}
$$

Material from Olary, South Australia. The data of Vaes and Kerr (1949), not cited below, probably were obtained on an impure sample. The water content of carnotite varies zeolitically between limits with attendant variation in the spacings. Carnotite is often intimately admixed with clayey material, quartz, tyuyamunite, or other impurities. The patterns are faint or diffuse due to small particle size or to mechanical deformation during grinding.

\begin{tabular}{|c|c|c|c|}
\hline$d(\mathrm{~A})$ & $I$ & $d(\mathrm{~A})$ & $I$ \\
\hline $\begin{array}{l}\text { 6. } 56 \\
\text { 5. } 12 \\
\text { 4. } 53 \\
\text { 4. } 25 \\
\text { 3. 53 } \\
\text { 3. } 25 \\
\text { 3. } 12 \\
\text { 2. } 715 \\
\text { 2. } 594 \\
\text { 2. } 571 \\
\text { 2. } 475 \\
\text { 2. } 156\end{array}$ & $\begin{array}{r}10 \\
1 \\
1 / 2 \\
3 \\
5 \\
3 \\
7 \\
1 \\
1 / 2 \\
2 \\
1 \\
3\end{array}$ & $\begin{array}{l}2.032 \\
1.998 \\
1.942 \\
1.914 \\
1.831 \\
1.784 \\
1.678 \\
1.655 \\
1.613 \\
1.531 \\
1.508 \\
1.455\end{array}$ & $\begin{array}{l}1 \\
1 \\
2 \\
1 \\
1 \\
1 \\
1 \\
1 \\
1 \\
1 \\
1 \\
1\end{array}$ \\
\hline
\end{tabular}

\section{CHERALITE}

$$
(\mathrm{Th}, \mathrm{Ca}, \mathrm{Ce}, \mathrm{La}, \mathrm{U}, \mathrm{Pb})\left(\mathrm{PO}_{4}, \mathrm{SiO}_{4}\right)
$$

Data from Bowie and Horne (1953) on material from Travancore,

\begin{tabular}{|c|c|c|c|}
\hline$d(\mathrm{~A})$ & $I$ & $d(\mathrm{~A})$ & $I$ \\
\hline $\begin{array}{l}\text { 5. } 20 \\
4.68 \\
\text { 4. } 14 \\
\text { 3. } 49 \\
\text { 3. } 26 \\
\text { 3. } 07 \\
\text { 2. } 96 \\
\text { 2. } 86 \\
\text { 2. } 60 \\
\text { 2. } 44\end{array}$ & 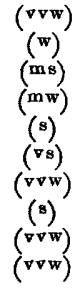 & $\begin{array}{l}\text { 2. } 18 \\
\text { 2. } 13 \\
\text { 1. } 958 \\
\text { 1. } 870 \\
\text { 1. } 795 \\
\text { 1. } 758 \\
\text { 1. } 733 \\
\text { 1. } 684 \\
\text { 1. } 534\end{array}$ & $\begin{array}{c}(\mathrm{mw}) \\
(\mathrm{mwb}) \\
(\mathrm{w}) \\
(\mathrm{mwb}) \\
(\mathrm{ww}) \\
(\mathrm{vww}) \\
(\mathrm{mw}) \\
(\mathrm{w}) \\
(\mathrm{ww})\end{array}$ \\
\hline $\begin{array}{l}\text { Very stro } \\
\text { Neak. } \\
\text { Very we } \\
\text { trong. }\end{array}$ & & \multicolumn{2}{|c|}{$\begin{array}{l}\text { mw Modprately weak. } \\
\text { mwb Moderately weak, broad. } \\
\text { ms Moderately strong. } \\
\text { vv } \text { w Very, very weak. }\end{array}$} \\
\hline
\end{tabular}
India. 


\section{CLARKEITE \\ $(\mathrm{Na}, \mathrm{K}, \mathrm{Ca}, \mathrm{Pb})_{2} \mathrm{U}_{2} \mathrm{O}_{7} \cdot n \mathrm{H}_{2} \mathrm{O}$}

Type material from Spruce Pine, N. C. See also Gruner (1954) and Frondel and Meyrowitz (1956). The composition of clarkeite apparently can vary widely, with accompanying variation in spacings, but detailed information is lacking.

\begin{tabular}{c|r||l|l}
\hline$d(\mathrm{~A})$ & $I$ & $d(\mathrm{~A})$ & $I$ \\
\hline 10.98 & 1 & 1.564 & 2 \\
8.33 & 1 & 1.537 & 1 \\
6.70 & 2 & 1.416 & 1 \\
.5 .77 & 8 & 1.389 & 1 \\
4.09 & 4 & 1.351 & 2 \\
3.34 & 9 & 1.278 & 4 \\
3.17 & 10 & 1.239 & 2 \\
2.92 & 3 & 1.227 & 1 \\
2.69 & 5 & 1.182 & 1 \\
2.61 & 4 & 1.152 & 1 \\
2.45 & 3 & 1.140 & 1 \\
2.06 & 2 & 1.118 & 2 \\
2.03 & 1 & 1.064 & 2 \\
1.968 & 7 & 1.044 & 2 \\
1.863 & 6 & .989 & 2 \\
1.764 & 5 & .975 & 1 \\
1.697 & 2 & .947 & 1 \\
1.676 & 3 & .937 & 1 \\
1.636 & 5 & .929 & 1 \\
1.589 & 2 & .917 & 1 \\
\hline
\end{tabular}

.bd_Broad, diffuse. 


\section{COFFINITE}

$$
\text { Ideally } \mathrm{U}\left(\mathrm{SiO}_{4}\right)_{1-\mathrm{x}}(\mathrm{OH})_{4 \mathrm{x}}
$$

Material from the Arrowhead mine, Gateway district, Mesa County, Colo. The pattern is similar to that of thorite. Nothing is known of the variation in spacings attending variation in content of $(\mathrm{OH})$ or of other substitutions in the cation or anion positions. The pattern of coffinite often is rather diffuse due to small particle size. Coffinite ordinarily is found virtually inextricably admixed with fine-grained uraninite. The indexing given below is for a tetragonal cell with $\mathrm{a}_{\mathrm{o}}=6.94, \mathrm{c}_{\mathrm{o}}=6.31 \mathrm{~A}$.

\begin{tabular}{c|c|c|c}
\hline$d$ (meas.) A & $I$ & $d$ (calc.) A & hkl \\
\cline { 2 - 3 } 4.66 & $(\mathrm{~s})$ & 4.66 & \\
3.47 & $(\mathrm{~s})$ & 3.47 & 011 \\
2.78 & $(\mathrm{w})$ & 2.78 & 121 \\
2.64 & $(\mathrm{~m})$ & 2.65 & 112 \\
2.46 & $(\mathrm{f})$ & 2.45 & 220 \\
2.18 & $(\mathrm{w})$ & 2.17 & 031 \\
2.01 & $(\mathrm{f})$ & 2.01 & 013 \\
1.841 & $(\mathrm{w})$ & 1.841 & 321 \\
1.801 & $(\mathrm{~m})$ & 1.801 & 312 \\
1.737 & $(\mathrm{w})$ & 1.740 & 123 \\
1.629 & $(\mathrm{f})$ & 1.735 & 400 \\
1.556 & $(\mathrm{f})$ & 1.626 & 411 \\
1.451 & $(\mathrm{f})$ & 1.552 & 420 \\
1.435 & $(\mathrm{f})$ & 1.435 & 332 \\
& & & 024 \\
\hline
\end{tabular}

- Strong. m Medium. $\quad$ Weak. 1 Faint. 


\section{CUPROSKLODOWSKITE \\ $\mathrm{Cu}\left(\mathrm{UO}_{2}\right)_{2}\left(\mathrm{SiO}_{3}\right)_{2}(\mathrm{OH})_{2} \cdot 5 \mathrm{H}_{2} \mathrm{O}$}

Material from Joachimsthal, Bohemia. Some types of 'cuprosklodowskite are extremely fine grained and give faint and'diffuse patterns. 4

\begin{tabular}{l|r||l|l}
\hline$d(\mathrm{~A})$ & $I$ & $d(\mathrm{~A})$ & $I$ \\
\hline 8.18 & 10 & 1.906 & $5^{\text {b }}$ \\
6.10 & 6 & 1.860 & $4^{\text {b }}$ \\
4.82 & 7 & 1.805 & 3 \\
4.29 & 2 & 1.766 & 3 \\
4.09 & 9 & 1.736 & 2 \\
3.52 & 6 & 1.703 & 1 \\
3.30 & 2 & 1.643 & 3 \\
3.20 & 2 & 1.614 & 1 \\
3.10 & 1 & 1.587 & 1 \\
2.97 & 8 & 1.564 & 1 \\
2.87 & 1 & 1.503 & 1 \\
2.72 & 5 & 1.472 & 2 \\
2.64 & 5 & 1.436 & 2 \\
2.36 & 1 & 1.397 & 1 \\
2.29 & 2 & 1.362 & 1 vb \\
2.21 & 6 & 1.332 & 1 \\
2.17 & 3 & 1.301 & 2 vd \\
2.12 & 2 & 1.279 & 2 \\
2.07 & 2 & 1.227 & 1 \\
2.04 & 4 & & \\
\hline
\end{tabular}

b Broad. vb Very broad. vd Very diffuse.

CURITE

$3 \mathrm{PbO} \cdot 8 \mathrm{UO}_{3} \cdot 4 \mathrm{H}_{2} \mathrm{O}$

Material from the Katanga district, Belgian Congo. The pattern of_curite is distinctive.

\begin{tabular}{l|r||l|l}
\hline$d(\mathrm{~A})$ & $I$ & $d(\mathrm{~A})$ & $I$ \\
\hline 6.28 & 10 & 1.95 & \\
4.17 & 1 & 1.91 & 1 \\
3.97 & 9 & 1.90 & 1 \\
3.53 & 4 & 1.87 & 1 \\
3.36 & 4 & 1.84 & 2 \\
3.14 & 8 & 1.80 & 3 \\
3.07 & 4 & 1.74 & 5 \\
2.93 & 2 & 1.70 & 2 \\
2.81 & 1 & 1.66 & 2 \\
2.70 & 4 & 1.62 & 2 \\
2.55 & 6 & 1.57 & 2 \\
2.47 & 3 & 1.54 & 1 \\
2.35 & 1 & 1.51 & 1 \\
2.24 & 1 & 1.49 & 1 \\
2.17 & 1 & 1.47 & 1 \\
2.10 & 5 & 1.43 & 1 \\
2.04 & 1 & 1.40 & 3 \\
2.00 & 1 & & \\
\hline
\end{tabular}




\section{DAVIDITE}

Near $\left(\mathrm{Fe}^{2}, \mathrm{U}, \mathrm{Ca}, \mathrm{Zr},{ }^{\top} \mathrm{Th}\right.$, rare-earths) $\left(\mathrm{Ti}, \mathrm{Fe}^{3}, \mathrm{~V}, \mathrm{Cr}\right)_{3}(\mathrm{O}, \mathrm{OH})_{7}$

Davidite apparently is always metamict. The data here cited, obtained by Bannister and Horne (1950) and Kerr and Holland (1951) on recrystallized metamict material from two localities, are not in close agreement due presumably to differences in chemical composition and the manner of heat treatment. Material heated at $1,000^{\circ} \mathrm{C}$ in air.

Kerr and Holland (1951)

\begin{tabular}{|c|c|c|c|}
\hline \multicolumn{2}{|c|}{$\begin{array}{l}\text { Copper radiation } \\
\text { Mozambique }\end{array}$} & \multicolumn{2}{|c|}{$\begin{array}{l}\text { Copper radiation } \\
\text { South Australia }\end{array}$} \\
\hline$d(\mathrm{~A})$ & $I$ & $d(\mathrm{~A})$ & $I$ \\
\hline $\begin{array}{l}\text { 6. } 71 \\
\text { 5. } 16 \\
\text { 4. } 47 \\
\text { 4. } 15 \\
3.40 \\
3.23 \\
\text { 3. } 05 \\
\text { 2. } 87 \\
\text { 2. } 62 \\
\text { 2. 48 } \\
\text { 2. 42 } \\
2.25 \\
2.19 \\
2.10 \\
2.03 \\
1.96 \\
1.91 \\
1.84 \\
1.80 \\
1.78 \\
1.70 \\
1.60 \\
1.57 \\
1.55 \\
1.51 \\
1.44 \\
1.38 \\
1.12 \\
1.07 \\
.90 \\
.89 \\
.86 \\
.83 \\
.82\end{array}$ & $\begin{array}{r}2 \\
1 \\
2 \\
6 \\
9 \\
8 \\
8 \\
10 \\
3 \\
8 \\
3 \\
8 \\
8 \\
3 \\
2 \\
1 \\
2 \\
4 \\
2 \\
6 \\
1 \\
9 \\
5 \\
5 \\
1 \\
1 \\
3 \\
7 \\
4 \\
4 \\
1 \\
2 \\
3 \\
2 \\
3 \\
3 \\
1\end{array}$ & $\begin{array}{l}3.42 \\
3.25 \\
3.08 \\
2.86 \\
2.65 \\
2.49 \\
2.44 \\
2.26 \\
2.20 \\
2.15 \\
2.09 \\
2.04 \\
1.97 \\
1.92 \\
1.85 \\
1.81 \\
1.78 \\
1.71 \\
1.69 \\
1.65 \\
1.60 \\
1.58 \\
1.55 \\
1.51 \\
1.44\end{array}$ & $\begin{array}{r}6 \\
6 \\
3 \\
10 \\
2 \\
7 \\
3 \\
7 \\
4 \\
1 \\
1 \\
4 \\
1 \\
2 \\
2 \\
8 \\
2 \\
8 \\
8 \\
1 \\
9 \\
2 \\
2 \\
1 \\
9\end{array}$ \\
\hline
\end{tabular}


Bannister and Horne (1950)

\begin{tabular}{|c|c|c|c|}
\hline \multicolumn{2}{|c|}{$\begin{array}{l}\text { Cobalt radiation } \\
\text { Mozambique }\end{array}$} & \multicolumn{2}{|c|}{$\begin{array}{l}\text { Cobalt radiation } \\
\text { South Australia }\end{array}$} \\
\hline$d(\mathrm{~A})$ & $I$ & $d(\mathrm{~A})$ & $I$ \\
\hline $\begin{array}{l}\text { 4. } 1 \\
\text { 3. } 64 \\
3.37 \\
\text { 3. } 21 \\
\text { 3. } 03 \\
\text { 2. } 96 \\
2.86 \\
\text { 2. } 82 \\
\text { 2. } 74 \\
\text { 2. } 62 \\
\text { 2. } 58 \\
\text { 2. } 49 \\
\text { 2. } 46 \\
2.41 \\
2.23 \\
2.18 \\
2.12 \\
1.91 \\
1.89 \\
1.80 \\
1.70 \\
1.69 \\
1.59 \\
1.54 \\
1.51 \\
1.435 \\
1.373 \\
1.364 \\
1.213 \\
1.153 \\
1.123\end{array}$ & $\begin{array}{r}5 \\
3 \\
7 \\
7 \\
6 \\
6 \\
8 \\
7 \\
4 \\
4 \\
4 \\
5 \\
5 \\
4 \\
8 \\
4 \\
7 \\
4 \\
4 \\
7 \\
10 \\
10 \\
8 \\
4 \\
4 \\
9 \\
7 \\
7 \\
6 \\
5 \\
6\end{array}$ & $\begin{array}{l}\text { 3. } 45 \\
3.41 \\
3.32 \\
3.22 \\
3.03 \\
2.97 \\
2.48 \\
2.46 \\
2.42 \\
2.24 \\
2.19 \\
2.13 \\
1.97 \\
1.90 \\
1.86 \\
1.80 \\
1.70 \\
1.68 \\
1.62 \\
1.60 \\
1.54 \\
1.436 \\
1.370 \\
1.357 \\
1.349 \\
1.096 \\
1.042\end{array}$ & $\begin{array}{r}5 \\
5 \\
5 \\
8 \\
2 \\
2 \\
6 \\
5 \\
4 \\
5 \\
5 \\
5 \\
2 \\
2 \\
5 \\
5 \\
3 \\
10 \\
4 \\
5 \\
4 \\
5 \\
3 \\
4 \\
4 \\
3 \\
6\end{array}$ \\
\hline
\end{tabular}




\section{DELORENZITE}

Near $(\mathrm{U}, \mathrm{Fe}, \mathrm{V})(\mathrm{Ti}, \mathrm{Sn})_{3} \mathrm{O}_{8}$

Data obtained on metamict delorenzite from the type locality, Craveggia, Piedmont, Italy, recrystallized by heating in air at $1000^{\circ} \mathrm{C}$.

\begin{tabular}{c|r||c|c}
\hline$d(\mathrm{~A})$ & $I$ & $d(\mathrm{~A})$ & $I$ \\
\hline 3.12 & 10 & 2.52 & 3 \\
2.87 & 7 & 2.28 & 1 \\
2.67 & 3 & 2.15 & 1 \\
\hline
\end{tabular}

\section{DEWINDTITE}

A hydrated lead uranyl phosphate

The status of dewindtite is uncertain. The X-ray pattern of apparently authentic material is very close to that of renardite, and the two minerals may be identical.

\section{DIDERICHITE}

Identical with rutherfordine.

$$
\begin{gathered}
\text { DUMONTITE } \\
\mathrm{Pb}_{2}\left(\mathrm{UO}_{2}\right)_{3}\left(\mathrm{PO}_{4}\right)_{2}(\mathrm{OH})_{4} \cdot 3 \mathrm{H}_{2} \mathrm{O}
\end{gathered}
$$

The specimen from the Katanga district, Belgian Congo, on which the present data were obtained is believed to be authentic but is not wholly beyond question.

\begin{tabular}{l|r||l|l}
\hline$d(\mathrm{~A})$ & \multicolumn{1}{|c||}{$I$} & $d(\mathrm{~A})$ & $I$ \\
\hline 7.69 & 4 & 2.58 & 1 \\
6.97 & 4 & 2.48 & 1 \\
6.11 & 4 & 2.15 & 3 \\
5.68 & 4 & 2.11 & 2 \\
4.29 & 10 & 2.04 & 2 \\
3.79 & 1 & 1.95 & 1 \\
3.75 & 9 & 1.93 & 2 \\
3.49 & 6 & 1.85 & 1 \\
3.35 & 2 & 1.77 & 1 \\
3.02 & 10 & 1.74 & 1 \\
2.96 & 5 & 1.65. & 1 \\
2.87 & 5 & 1.52 & 1 \\
2.69 & 2 & & \\
\hline
\end{tabular}




\section{EPI-IANTHINITE}

\section{Near $\mathrm{UO}_{3} \cdot 2 \mathrm{H}_{2} \mathrm{O}(?)$}

X-ray powder data for authentic or type epi-ianthinite are lacking. Data have been published, however, by J. W. Frondel and Cuttitta (1954) for an alteration product of ianthinite that ${ }_{4}$ very probably represents epi-ianthinite. These data are given below.

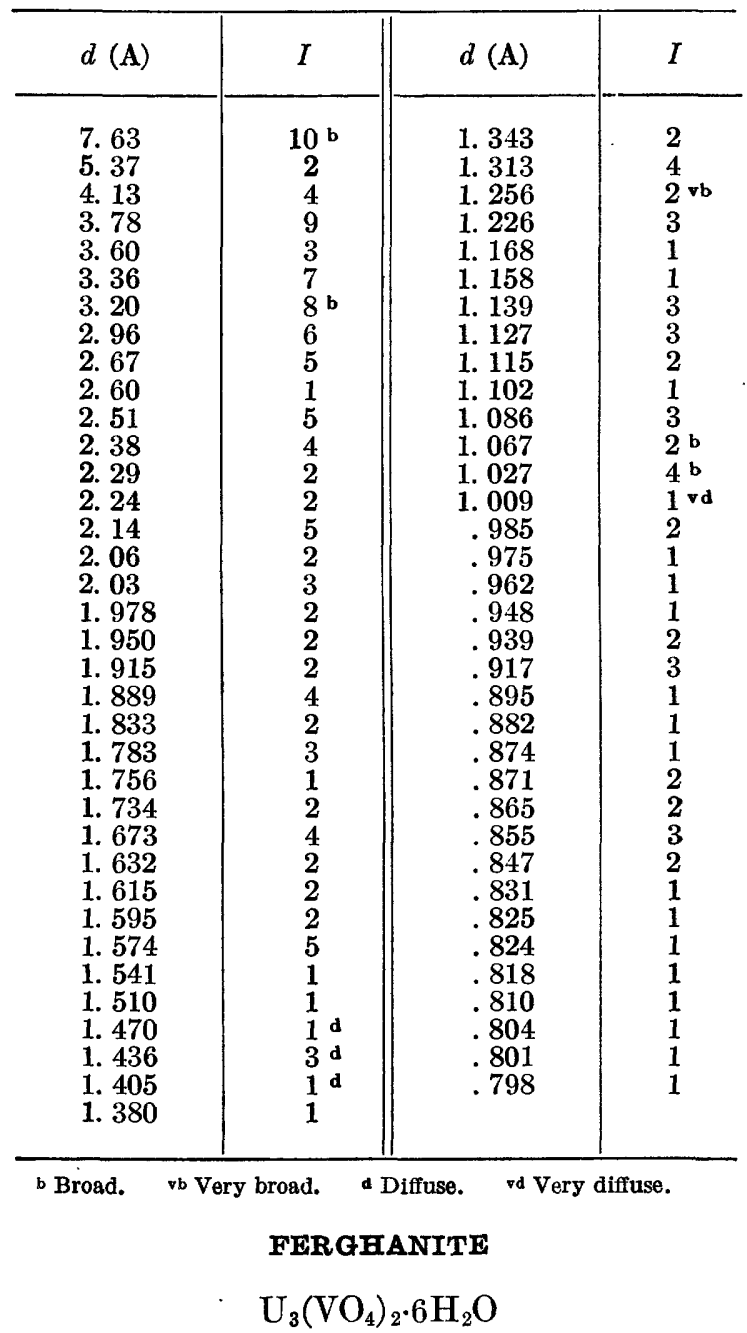

X-ray powder spacing data are lacking. 


\section{FOURMARIERITE}

\section{$\mathrm{PbO} \cdot 4 \mathrm{UO}_{3} \cdot 7 \mathrm{H}_{2} \mathrm{O}$}

Material from Wölsendorf, Bavaria. The X-ray pattern is slightly variable and, in general, is similar to that of synthetic hydrated lead diuranate and of synthetic solid solutions extending from that composition to lower lead contents. Some natural material, whose proper species designation is uncertain, has a pattern identical with that of hydrated lead diuranate (for which data are given below). There also is a more or less close resemblance in pattern to vandendriesscheite, masuyite, and schoepite, and these minerals are at least closely related in structure-if indeed, a continuous series does not extend between them. The patterns of all of these minerals are distinguished by a pair of very dark lines with $d=3.4-3.6$ and $d=3.0-3.2$, the values apparently increasing with increasing content of lead. Strong and sharp patterns, difficult to obtain with these minerals, are needed for positive identification. Faint patterns are ambiguous and supplementary optical and chemical tests are needed. (See Frondel, 1956.)

\begin{tabular}{|c|c|c|c|c|c|}
\hline \multicolumn{4}{|c|}{ Wölsendorf, Bavaria } & \multicolumn{2}{|c|}{$\begin{array}{c}\text { Synthetic hydrated lead } \\
\text { diuranate }\end{array}$} \\
\hline$d(\mathrm{~A})$ & $I$ & $d(\mathrm{~A})$ & $I$ & $d(\mathrm{~A})$ & $I$ \\
\hline $\begin{array}{l}7.86 \\
6.83 \\
3.45 \\
3.09 \\
2.73 \\
2.44 \\
2.26 \\
2.15 \\
2.09 \\
1.996 \\
1.907 \\
1.817 \\
1.729 \\
1.687 \\
1.669 \\
1.645 \\
1.607 \\
1.544 \\
1.508\end{array}$ & $\begin{array}{r}2 \\
4 \\
9 \\
10 \\
4 \\
6 \\
5 \\
2 \\
2 \\
6 \\
8 \\
2 \\
7 \\
2 \\
2 \\
3 \\
2 \\
3 \\
1\end{array}$ & $\begin{array}{r}1.486 \\
1.376 \\
1.346 \\
1.309 \\
1.284 \\
1.252 \\
1.226 \\
1.192 \\
1.177 \\
1.142 \\
1.127 \\
1.114 \\
1.102 \\
1.070 \\
1.055 \\
1.030 \\
1.012 \\
.994 \\
.974 \\
.945\end{array}$ & $\begin{array}{l}2 \\
2 \\
3 \\
2 \\
2 \\
1 \\
1 \\
1 \\
2 \\
1 \\
1 \\
1 \\
1 \\
2 \\
1 \\
1 \\
1 \\
1 \\
1 \\
2\end{array}$ & $\begin{array}{l}6.81 \\
3.43 \\
3.08 \\
2.755 \\
2.436 \\
2.270 \\
1.990 \\
1.903 \\
1.811 \\
1.719 \\
1.672 \\
1.535 \\
1.497 \\
1.377 \\
1.342 \\
1.299 \\
1.276 \\
1.217 \\
1.132\end{array}$ & $\begin{array}{r}3 \\
9 \\
10 \\
1 \\
3 \\
2 \\
6 \\
7 \\
1 \\
6 \\
4 \\
3 \\
2 \\
3 \\
2 \\
4 \\
5 \\
3 \\
4\end{array}$ \\
\hline
\end{tabular}




\section{FRITZCHEITE}

A hydrated manganese uranyl phosphate-vanadate

Doubtful species for which X-ray powder data are lacking.

\section{GUMMITE}

A generic term, not a species name, formerly used to designate finegrained yellow to orange and orange-red uranium minerals, chiefly hydrated lead uranyl oxides pseudomorphous after uraninite, whose true identity was unknown. (See Frondel, 1956.)

\section{HUTTONITE}

\section{$\mathrm{ThSiO}_{4}$}

Data from Pabst (1951) on natural material from South Westland, New Zealand. The pattern of huttonite is quite distinct from that of its polymorph thorite but similar to that of monazite.

\begin{tabular}{l|l||l|l}
\hline$d(\mathrm{~A})$ & $I$ & $d(\mathrm{~A})$ & $I$ \\
\hline 5.29 & 3 & 2.16 & 2 \\
4.71 & 5 & 2.11 & 3 \\
4.23 & 6 & 1.953 & 4 \\
4.08 & 4 & 1.893 & $3 \mathrm{~b}$ \\
3.53 & 4 & 1.857 & $2 \mathrm{~b}$ \\
3.29 & 6 & 1.810 & 2 \\
3.09 & 8 & 1.784 & 2 \\
2.98 & 3 & 1.749 & $4 \mathrm{~b}$ \\
2.89 & 7 & 1.692 & 2 \\
2.65 & 3 & 1.646 & 2 \\
2.48 & 3 & 1.603 & 3 \\
2.44 & 1 & 1.550 & 3 \\
2.19 & 4 & & \\
\hline
\end{tabular}

- Broad. 


\section{HYDROGEN-AUTUNITE}

$$
\mathrm{H}_{2}\left(\mathrm{UO}_{2}\right)_{2}\left(\mathrm{PO}_{4}\right)_{2} \cdot 8 \mathrm{H}_{2} \mathrm{O}
$$

The hydrogen analogue of meta-autunite has been synthesized and there is some evidence that either it or an intermediate member in a series to meta-autunite occurs in nature. The data given below, from Ross (1955a), is for synthetic material with $8 \mathrm{H}_{2} \mathrm{O}$.

\begin{tabular}{|c|c|c|c|c|c|}
\hline$d(\mathbf{A})$ & $I$ & hkl & $d(\mathbf{A})$ & $I$ & hkl \\
\hline $\begin{array}{l}9.032 \\
5.556 \\
\text { 4. } 971 \\
\text { 4. } 542 \\
\text { 4. } 360 \\
\text { 3. } 799 \\
\text { 3. } 511 \\
\text { 3. } 270 \\
2.964 \\
2.765 \\
2.576 \\
2.488 \\
2.397 \\
2.267 \\
2.216 \\
2.163 \\
2.075 \\
1.902 \\
1.844\end{array}$ & $\begin{array}{r}10 \\
5 \\
4 \\
1 / 2 \\
3 \\
9 \\
7 \\
8 \\
6 \\
7 \\
3 \\
3 \\
4 \\
2 \\
3 \\
5 \\
4 \\
2 \\
3\end{array}$ & $\begin{array}{l}001 \\
011 \\
110 \\
002 \\
111 \\
102 \\
200 \\
021 \\
121 \\
022,103 \\
122,113 \\
220 \\
221 \\
301,004 \\
310 \\
311,104 \\
302 \\
321,204 \\
303\end{array}$ & $\begin{array}{l}\text { 1. } 789 \\
1.755 \\
1.722 \\
1.697 \\
1.633 \\
1.61-1.57 \\
1.546 \\
1.477 \\
1.439 \\
1.401 \\
1.383 \\
1.359 \\
1.338 \\
1.288 \\
1.270 \\
1.249 \\
1.221 \\
1.194\end{array}$ & $\begin{array}{l}3 \\
2 \\
2 \\
3 \\
3 \\
3 \\
1 \\
1 \\
1 \\
2 \\
1 \\
1 \\
1 / 2 \\
1 / 2 \\
1 \\
2 \\
1 / 2 \\
3\end{array}$ & $\begin{array}{l}322,313 \\
400,105 \\
401 \\
115 \\
402,323 \\
314, \text { etc. } \\
421 \\
324,106 \\
116 \\
315 \\
206,511 \\
414,216 \\
334 \\
424,226 \\
107,306 \\
316 \\
335 \\
531,504,326\end{array}$ \\
\hline
\end{tabular}

b Broad. 


\section{IANTHINITE}

A hydrated oxide of quadrivalent uranium

The identity and composition of the several violet minerals and synthetic preparations that have been referred to the name ianthinite are uncertain. The following data from Bignand (1955) refer to (a) a violet hydrated uranous oxide, known both as a natural mineral and as a synthetic product, that may correspond to the original ianthinite of Schoep, and (b) a violet mineral containing both quadrivalent and sexivalent uranium with calcium carbonate and water.

\begin{tabular}{|c|c|c|c|}
\hline \multicolumn{2}{|c|}{ (a) Ianthinite? } & \multicolumn{2}{|c|}{$\begin{array}{l}\text { (b) Uranium calcium } \\
\text { carbonate }\end{array}$} \\
\hline$d(\mathrm{~A})$ & $I$ & $d(\mathrm{~A})$ & $I$ \\
\hline $\begin{array}{l}7.60 \\
3.79 \\
3.59 \\
3.35 \\
3.28 \\
2.96 \\
2.89 \\
2.68 \\
2.61 \\
2.53 \\
2.38 \\
2.25 \\
2.14 \\
2.06 \\
2.02 \\
1.98 \\
1.95 \\
1.92 \\
1.89 \\
1.83 \\
1.79 \\
1.74 \\
1.67 \\
1.64 \\
1.34 \\
1.32\end{array}$ & $\begin{array}{c}(\mathrm{vs}) \\
(\mathrm{ms}) \\
(\mathrm{ms}) \\
(\mathrm{ms}) \\
(\mathrm{m}) \\
(\mathrm{mf}) \\
(\mathrm{mf}) \\
(\mathrm{f}) \\
(\mathrm{mf}) \\
(\mathrm{mf}) \\
(\mathrm{f}) \\
(\mathrm{f}) \\
(\mathrm{f}) \\
(\mathrm{f}) \\
(\mathrm{mf}) \\
(f) \\
(f) \\
(f) \\
(\mathrm{ff}) \\
(\mathrm{f}) \\
(\mathrm{mf}) \\
(\mathrm{mf}) \\
(\mathrm{ms}) \\
(\mathrm{f}) \\
(\mathrm{mf}) \\
(\mathrm{mf})\end{array}$ & $\begin{array}{r}10.29 \\
7.60 \\
5.19 \\
4.07 \\
3.35 \\
3.28 \\
3.01 \\
2.59 \\
2.52 \\
2.47 \\
2.20 \\
2.03 \\
2.00 \\
1.96 \\
1.77 \\
1.73 \\
1.66 \\
1.57 \\
1.53 \\
1.50\end{array}$ & $\begin{array}{l}(\mathrm{vs}) \\
(\mathrm{vf}) \\
(\mathrm{ms}) \\
(\mathrm{m}) \\
(f) \\
(\mathrm{mg}) \\
(\mathrm{m}) \\
(\mathrm{m}) \\
(\mathrm{f}) \\
(f) \\
(f) \\
(\mathrm{mf}) \\
(\mathrm{vf}) \\
(\mathrm{vf}) \\
(f) \\
(f) \\
(f) \\
(f) \\
(f) \\
(\mathrm{f}) \\
(\mathrm{vf})\end{array}$ \\
\hline
\end{tabular}




$$
\begin{gathered}
\text { JOHANNITE } \\
\mathrm{Cu}\left(\mathrm{UO}_{2}\right)_{2}\left(\mathrm{SO}_{4}\right)_{2}(\mathrm{OH})_{2} \cdot 6 \mathrm{H}_{2} \mathrm{O}
\end{gathered}
$$

Material from Great Bear Lake, Northwest Territory, Canada.

\begin{tabular}{|c|c|c|c|}
\hline$d(\mathrm{~A})$ & $I$ & $d(\mathbf{A})$ & $I$ \\
\hline $\begin{array}{l}7.73 \\
6.16 \\
\text { 5. } 59 \\
4.84 \\
4.38 \\
\text { 4. } 20 \\
\text { 3. } 87 \\
\text { 3. } 73 \\
\text { 3. } 53 \\
\text { 3. } 41 \\
\text { 3. } 13 \\
\text { 3. } 04 \\
2.93 \\
2.81 \\
2.69 \\
2.58 \\
2.45 \\
2.37 \\
2.23 \\
2.19 \\
2.14 \\
2.09 \\
2.01 \\
1.925 \\
1.898 \\
1.844 \\
1.798\end{array}$ & $\begin{array}{r}10 \\
9 \\
4 \\
2 \\
6 \\
3 \\
7 \\
4 \\
4 \\
8 \\
7 \\
7 \\
2 \\
3 \\
4 \\
3 \\
2 \\
1 \\
2 \\
1 \\
3 \\
2 \\
2 \\
2 \\
1 \\
3 \\
1\end{array}$ & $\begin{array}{r}1.764 \\
1.708 \\
1.669 \\
1.591 \\
1.565 \\
1.565 \\
1.549 \\
1.525 \\
1.459 \\
1.433 \\
1.411 \\
1.375 \\
1.351 \\
1.285 \\
1.196 \\
1.180 \\
1.159 \\
1.133 \\
1.120 \\
1.104 \\
1.087 \\
1.066 \\
1.041 \\
.931 \\
.920 \\
.839\end{array}$ & $\begin{array}{l}2 \\
2 \\
1 \\
2 \\
1 \\
1 \\
1 \\
2 \\
1 \\
1 \\
1 \\
1 \\
1 \\
1 \\
2 \\
2 \\
1 \\
2 \\
2 \\
1 \\
1 \\
1 \\
1 \\
1 \\
1 \\
1\end{array}$ \\
\hline
\end{tabular}
Identical patterns are given by material from other localities.

\section{KAHLERITE}

$$
\mathrm{Fe}\left(\mathrm{UO}_{2}\right)_{2}\left(\mathrm{AsO}_{4}\right)_{2} .8 \mathrm{H}_{2} \mathrm{O}
$$

$\mathrm{X}$-ray powder data for kahlerite are lacking. It is not certain whether kahlerite belongs in the torbernite or the metatorbernite group; the pattern should closely resemble that of bassetite or zeunerite in the former group or that of metazeunerite in the latter. 
KASOLITE

\section{$\mathrm{Pb}\left(\mathrm{UO}_{2}\right)\left(\mathrm{SiO}_{3}\right)(\mathrm{OH})_{2}$}

Material from Shinkolobwe, Katanga, Belgian Congo. Specimens with the same optical properties from other localities give an identical pattern. Material from a few localities shows diminished indices of refraction and contains more or less $\mathrm{Ca}$ in substitution for $\mathrm{Pb}$ (Mineral B of Frondel, 1956); the X-ray pattern is virtually identical with that of ordinary kasolite.

\begin{tabular}{l|l||l|l}
\hline$d(\mathrm{~A})$ & $I$ & $d(\mathrm{~A})$ & $I$ \\
\hline 6.61 & & & \\
6.19 & 6 & 1.397 & 1 \\
5.31 & 2 & 1.366 & 2 \\
4.76 & 4 & 1.329 & 2 \\
4.19 & 2 & 1.300 & 2 \\
3.53 & $8 \mathrm{~b}$ & 1.277 & 1 \\
3.38 & 7 & 1.262 & 1 \\
3.26 & 1 & 1.247 & 1 \\
3.07 & 10 & 1.235 & 1 \\
2.93 & 5 & 1.200 & 1 \\
2.73 & 9 & 1.183 & 1 \\
2.64 & 3 & 1.147 & 2 \\
2.47 & 3 & 1.114 & 2 \\
2.42 & 3 & 1.086 & 1 \\
2.37 & 2 & 1.049 & 1 \\
2.18 & 2 & 1.035 & 1 \\
2.11 & 3 & 1.017 & 1 \\
2.05 & 2 & 1.004 & 1 \\
1.962 & 3 & .993 & 1 \\
1.913 & 5 & .976 & 1 \\
1.876 & 1 & .969 & 1 \\
1.847 & 2 & .949 & 1 \\
1.826 & 1 & .943 & 1 \\
1.741 & 1 & .930 & 1 \\
1.677 & 4 & .920 & 1 \\
1.656 & 5 & .909 & 1 \\
1.623 & 2 & .899 & 1 \\
1.592 & 1 & .877 & 2 \\
1.565 & 1 & .870 & 1 \\
1.532 & 1 & .838 & 2 \\
1.497 & 1 & .831 & 1 \\
1.476 & 1 & .809 & 2 \\
1.456 & 2 & .803 & 1 \\
\hline & 3 & & \\
\hline & & & \\
\hline
\end{tabular}

b Broad. 


\section{LIEBIGITE}

$$
\mathrm{Ca}_{2}\left(\mathrm{UO}_{2}\right)\left(\mathrm{CO}_{3}\right)_{3} \cdot 10 \mathrm{H}_{2} \mathrm{O}
$$

Material from Joachimsthal, Bohemia. Slightly different values are given by Evans and Frondel (1950).

\begin{tabular}{|c|c|c|c|}
\hline$d(\mathrm{~A})$ & $I$ & $d(\mathrm{~A})$ & $I$ \\
\hline $\begin{array}{l}8.68 \\
8.27 \\
6.81 \\
6.11 \\
5.40 \\
4.95 \\
\text { 4. 55 } \\
\text { 4. } 17 \\
\text { 4. } 04 \\
\text { 3. } 95 \\
\text { 3. } 75 \\
3.58 \\
3.33 \\
3.31 \\
3.19 \\
3.10 \\
3.02 \\
2.84 \\
2.77 \\
2.66 \\
2.57 \\
2.44\end{array}$ & $\begin{array}{r}9 \\
1 \\
10 \\
2 \\
9 \\
1 \\
6 \\
1 \\
2 \\
1 \\
2 \\
3 \\
5 \\
5 \\
1 \\
6 \\
1 \\
1 \\
1 \\
1 \\
3 \\
1\end{array}$ & $\begin{array}{l}2.37 \\
2.30 \\
2.26 \\
2.15 \\
2.10 \\
2.02 \\
1.998 \\
1.957 \\
1.911 \\
1.884 \\
1.830 \\
1.716 \\
1.670 \\
1.561 \\
1.533 \\
1.484 \\
1.473 \\
1.437 \\
1.416 \\
1.397 \\
1.347\end{array}$ & $\begin{array}{l}2 \\
2 \\
2 \\
4 \\
1 \\
1 \\
5 \\
1 \\
1 \\
1 \\
1\end{array}$ \\
\hline
\end{tabular}

\section{MASUYITE}

$\mathrm{UO}_{3} \cdot 2 \mathrm{H}_{2} \mathrm{O}$

Material from the Katanga district, Belgian Congo. See further under fourmarierite.

\begin{tabular}{l|r||l|l}
\hline$d(\mathrm{~A})$ & $I$ & $d(\mathrm{~A})$ & $I$ \\
\hline 8.53 & 1 & 1.984 & 6 \\
7.10 & 10 & 1.908 & 3 \\
6.43 & 2 & 1.784 & $4^{\mathrm{d}}$ \\
4.80 & 1 & 1.727 & 2 \\
4.35 & 4 & 1.697 & 1 \\
3.92 & 2 & 1.662 & 1 \\
3.54 & 8 & 1.598 & 2 \\
3.15 & 9 & 1.510 & 2 \\
2.97 & 1 & 1.421 & $1^{\mathrm{d}}$ \\
2.74 & 3 & 1.380 & $1^{\text {vd }}$ \\
2.51 & 5 & 1.349 & 2 \\
2.38 & 2 & 1.329 & 2 \\
2.06 & 1 & & \\
\hline
\end{tabular}

d Diffuse. $\quad$ rd Very diffuse. 


\section{META-AUTUNITE}

$$
\mathrm{Ca}\left(\mathrm{UO}_{2}\right)_{2}\left(\mathrm{PO}_{4}\right)_{2} \cdot 6-8 \mathrm{H}_{2} \mathrm{O}
$$

Material from Sabugal, Portugal. The pattern of meta-autunite differs distinctly from those of the members of the fully hydrated torbernite group but is close to that of meta-uranocircite in the metatorbernite group. The transition between meta-autunite and autunite is reversible. The formation of meta-autunite is favored by increased temperature and reduced humidity and is the phase stable at ordinary conditions of temperature and pressure.

\begin{tabular}{|c|c|c|c|}
\hline$d(\mathrm{~A})$ & $I$ & $d(\mathrm{~A})$ & $I$ \\
\hline $\begin{array}{l}8.51 \\
5.39 \\
4.96 \\
4.28 \\
\text { 3. } 63 \\
\text { 3. 50 } \\
3.24 \\
2.94 \\
2.68 \\
2.61 \\
2.51 \\
2.47 \\
2.38 \\
2.25 \\
2.21 \\
2.14 \\
2.10 \\
2.04 \\
1.941 \\
1.893\end{array}$ & $\begin{array}{r}10 \\
7 \\
5 \\
6 \\
8 \\
9 \\
8 \\
4 \\
1 \\
3 \\
2 \\
2 \\
3 \\
2 \\
3 \\
3 \\
3 \\
3 \\
2 \\
1\end{array}$ & $\begin{array}{l}1.802 \\
1.757 \\
1.711 \\
1.658 \\
1.599 \\
1.568 \\
1.529 \\
1.454 \\
1.379 \\
1.346 \\
1.328 \\
1.283 \\
1.254 \\
1.186 \\
1.166 \\
1.153 \\
1.126 \\
1.109 \\
1.096 \\
1.065\end{array}$ & $\begin{array}{l}2 \\
3 \\
2 \\
2 \\
1 \\
4 \\
2 \\
2 \\
3{ }^{b} \\
2 \\
1 \\
2 \\
2 \\
1 \\
1 \\
1 \\
1 \\
1 \\
1 \\
1 \\
1 \\
1 \\
1\end{array}$ \\
\hline
\end{tabular}

b Broad. 


\section{METATORBERNITE}

\section{$\mathrm{Cu}\left(\mathrm{UO}_{2}\right)_{2}\left(\mathrm{PO}_{4}\right)_{2} \cdot 6-8 \mathrm{H}_{2} \mathrm{O}$}

Material from Gunnis Lake, Calstock, Cornwall. Direct comparison of films of this mineral from many different localities has shown little or no significant variation in spacings. The pattern of metatorbernite differs distinctly from those of the members of the fully hydrated torbernite group. It is similar to those of the other members of the metatorbernite group and is particularly close to that of metazeunerite. Confirmatory chemical tests are desirable.

\begin{tabular}{|c|c|c|c|}
\hline$d(\mathrm{~A})$ & $I$ & $d(\mathrm{~A})$ & $I$ \\
\hline 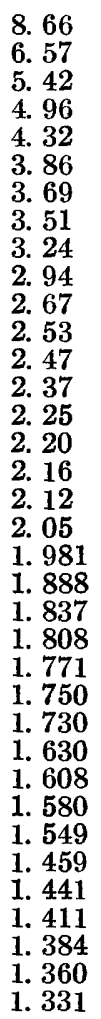 & $\begin{array}{r}9 \\
1 \\
6 \\
6 \\
5 \\
1 \\
10 \\
7 \\
8 \\
3 \\
4 \\
3 \\
1 \\
2 \\
1 \\
2 \\
3 \\
2 \\
3 \\
3 \\
1 \\
2 \\
1 \\
2 \\
1 \\
1 \\
3 \\
1 \\
2 \\
4 \\
1 \\
2 \\
3 \\
2 \\
3 \\
2\end{array}$ & $\begin{array}{l}1.310 \\
1.261 \\
1.227 \\
1.217 \\
1.207 \\
1.195 \\
1.181 \\
1.159 \\
1.151 \\
1.109 \\
1.094 \\
1.083 \\
1.070 \\
1.057 \\
1.044 \\
1.035 \\
1.022 \\
1.004 \\
.982 \\
.964 \\
.945 \\
.928 \\
.919 \\
.910 \\
.897 \\
.883 \\
.867 \\
.855 \\
.842 \\
.832 \\
.830 \\
.820 \\
.807 \\
.792 \\
.788 \\
.787\end{array}$ & $\begin{array}{l}2 \\
1 \\
1 \\
1 \\
1 \\
1 \\
1 \\
1 \\
2 \\
2 \\
1 \\
1 \\
1 \\
2 \\
1 \\
1 \\
1 \\
2 \\
1 \\
2 \\
1 \\
2 \\
2 \\
1 \\
1 \\
2 \\
1 \\
1 \\
1 \\
2 \\
1 \\
1 \\
1 \\
1 \\
1 \\
2 \\
1\end{array}$ \\
\hline
\end{tabular}

d Diffuse. 


\section{METATYUYAMUNITE \\ $\mathrm{Ca}\left(\mathrm{UO}_{2}\right)_{2}\left(\mathrm{VO}_{4}\right)_{2} \cdot 3-5 \mathrm{H}_{2} \mathrm{O}$}

Data from Stern and others (1956) on synthetic material and natural material from the Small Spot mine, Mesa County, Colo.; also data from the Harvard file on natural material from Ferghana, Russia. The water content varies zeolitically over the range 3 to $5 \mathrm{H}_{2} \mathrm{O}$ with attendant variation in the spacing of (001) and other planes; the (001) spacings decrease as the water content decreases. The patterns in general are of poor quality. Natural metatyuyamunite is more or less admixed with the higher hydrate, tyuyamunite, and lines of this phase may be present in the patterns.

\begin{tabular}{|c|c|c|c|c|c|}
\hline \multicolumn{2}{|c|}{ Synthetic } & \multicolumn{2}{|c|}{ Colorado } & \multicolumn{2}{|c|}{ Russia } \\
\hline$d(\mathrm{~A})$ & $I$ & $d(\mathrm{~A})$ & $I$ & $d(\mathrm{~A})$ & $I$ \\
\hline $\begin{array}{l}9.94 \\
8.51 \\
6.51 \\
5.19 \\
4.48 \\
4.22 \\
3.77 \\
3.55 \\
3.24 \\
3.04 \\
2.85 \\
2.72 \\
2.59 \\
2.48 \\
2.19 \\
2.13 \\
2.05 \\
2.01 \\
1.96 \\
1.91 \\
1.85 \\
1.77 \\
1.74 \\
1.69 \\
1.66 \\
1.62 \\
1.57 \\
1.53 \\
1.49\end{array}$ & 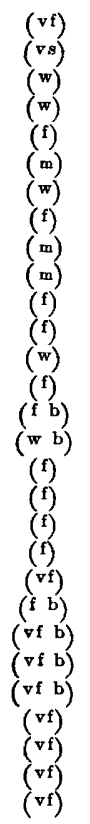 & $\begin{array}{l}8.51 \\
6.56 \\
5.19 \\
4.48 \\
4.22 \\
3.77 \\
3.58 \\
3.26 \\
3.05 \\
2.81 \\
2.58 \\
2.50 \\
2.35 \\
2.17 \\
2.12 \\
2.05 \\
1.99 \\
1.95 \\
1.91 \\
1.88 \\
1.85 \\
1.77 \\
1.73 \\
1.69 \\
1.66 \\
1.57 \\
1.53 \\
1.50\end{array}$ & $\begin{array}{l}(\mathrm{vs}) \\
(f) \\
(\mathrm{w}) \\
(f) \\
(\mathrm{s}) \\
(\mathrm{w}) \\
(f) \\
(\mathrm{fb}) \\
(\mathrm{mb}) \\
(\mathrm{w}) \\
(\mathrm{w}) \\
(\mathrm{w}) \\
(\mathrm{vf}) \\
(\mathrm{vf}) \\
(\mathrm{vf}) \\
(\mathrm{w} b) \\
(f) \\
(f) \\
(f) \\
(f) \\
(f) \\
(f) \\
(f) \\
(f) \\
(f) \\
(f) \\
(f) \\
(\mathrm{vf}) \\
(\mathrm{ff}) \\
(\mathrm{ff})\end{array}$ & $\begin{array}{l}8.43 \\
5.18 \\
4.46 \\
\text { 4. } 17 \\
3.74 \\
3.52 \\
3.28 \\
3.03 \\
2.805 \\
2.578 \\
2.113 \\
2.002 \\
1.949 \\
1.771\end{array}$ & $\begin{array}{r}10 \\
3 \\
1 \\
8 \\
2 \\
1 \\
6 \\
5 \\
2 \\
3 \\
4 \\
1 \\
1 \\
1\end{array}$ \\
\hline
\end{tabular}


X-RAY POWDER DATA FOR URANIUM AND THORIUM MINERALS 123

META-URANOCIRCITE

$\mathrm{Ba}\left(\mathrm{UO}_{2}\right)_{2}\left(\mathrm{PO}_{4}\right)_{2} \cdot 8 \mathrm{H}_{2} \mathrm{O}$

Data from Nuffield and Milne (1953) on natural material from Falkenstein, Saxony.

\begin{tabular}{l|r|r||c|c|c}
\hline$d(\mathrm{~A})$ & $I$ & hkl & $d(\mathrm{~A})$ & $I$ & hkl \\
\hline 8.19 & 7 & 002 & 2.18 & 2 & 130,026 \\
5.37 & 6 & 012 & 2.12 & 1 & 132,224 \\
4.90 & 3 & 110 & 2.08 & 7 & 126 \\
4.21 & 6 & 004 & 2.01 & 4 & 018 \\
3.58 & 10 & 014 & 1.924 & 3 & \\
3.39 & 2 & 021 & 1.883 & $1 / 2$ & \\
3.21 & 5 & 022 & 1.796 & 3 & \\
2.91 & 3 & 122 & 1.741 & 3 & \\
2.67 & $1 / 2$ & 024 & 1.676 & $1 / 2$ & \\
2.59 & 4 & 016 & 1.589 & 5 & \\
2.41 & $1 / 2$ & 025 & 1.559 & 1 & \\
2.35 & 1 & 222 & 1.518 & 5 & \\
\hline
\end{tabular}




\section{METAZEUNERITE \\ $\mathrm{CU}\left(\mathrm{UO}_{2}\right)_{2}\left(\mathrm{AsO}_{4}\right)_{2} \cdot 6-8 \mathrm{H}_{2} \mathrm{O}$}

Data from J. W. Frondel (1951) on synthetic material and natural material from Eureka, Utah, both with $8 \mathrm{H}_{2} \mathrm{O}$.

\begin{tabular}{l|l|l}
\hline \multicolumn{2}{c}{ Synthetic } & \\
\hline
\end{tabular}




\section{MICROLITE-PYROCHLORE}

$$
(\mathrm{Na}, \mathrm{Ca}, \mathrm{Fe}, \mathrm{U})_{2}(\mathrm{Ta}, \mathrm{Nb})_{2} \mathrm{O}_{6}(\mathrm{O}, \mathrm{OH}, \mathrm{F})
$$

Data of Arnott (1950), obtained on ignited microlite from Amelia Court House, Va. The spacings vary slightly attending the substitution of $\mathrm{U}, \mathrm{Nb}$, and other elements in the microlite-pyrochlore series. The members of the series, particularly those high in uranium, are often metamict.

\begin{tabular}{|c|c|c|c|c|c|}
\hline$d(\mathrm{~A})$ & $I$ & $\mathrm{hkl}$ & $d(\mathrm{~A})$ & $I$ & hkl \\
\hline 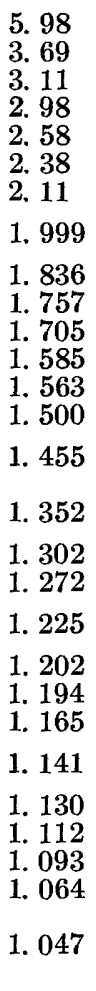 & $\begin{array}{r}6 \\
1 / 4 \\
4 \\
10 \\
3 \\
1 \\
1 / 4 \\
2 \\
8 \\
2 \\
1 / 2 \\
1 / 4 \\
8 \\
2 \\
2 \\
\\
2 \\
2 \\
1 / 2 \\
1 / 4 \\
1 / 4 \\
3 \\
3 \\
1 \\
1 / 4 \\
1 / 2 \\
1 \\
3\end{array}$ & $\begin{array}{l}111 \\
022 \\
113 \\
222 \\
004 \\
133 \\
224 \\
333 \\
115 \\
044 \\
135 \\
244 \\
335 \\
226 \\
444 \\
117 \\
155 \\
137 \\
355 \\
008 \\
337 \\
066 \\
228 \\
555 \\
266 \\
048 \\
119 \\
357 \\
248 \\
466 \\
139 \\
448 \\
177 \\
339 \\
557\end{array}$ & $\begin{array}{r}1.018 \\
1.009 \\
1.003 \\
.972 \\
.939 \\
.921 \\
.911 \\
.881 \\
.869 \\
.859 \\
.848 \\
.837 \\
.824 \\
.822 \\
.797 \\
.795 \\
.785 \\
.778 \\
.777\end{array}$ & $\begin{array}{c}1 / 2 \\
1 / 2 \\
3 \\
1 / 2 \\
1 \\
2 \\
2 \\
\\
4 \\
3 \\
1 \\
1 / 4 \\
2 \\
3 \\
1 \\
1\end{array}$ & $\left\{\begin{array}{l}0.2 .10 \\
268 \\
377 \\
159 \\
666 \\
359 \\
1.1 .11 \\
577 \\
088 \\
1.3 .11 \\
179 \\
559 \\
2.6 .10 \\
668 \\
0.0 .12 \\
488 \\
1.5 .11 \\
777 \\
2.2 .12 \\
4.6 .10 \\
3.5 .11 \\
579 \\
0.4 .12 \\
199 \\
1.1 .13 \\
1.7 .11 \\
5.5 .11 \\
399 \\
6.6 .10 \\
4.4 .12 \\
1.3 .13 \\
3.7 .11 \\
779 \\
0.6 .12 \\
4.8 .10\end{array}\right.$ \\
\hline
\end{tabular}




\section{NOVACEKITE \\ $\mathrm{Mg}\left(\mathrm{UO}_{2}\right)_{2}\left(\mathrm{AsO}_{4}\right)_{2} \cdot 8-10 \mathrm{H}_{2} \mathrm{O}$}

Material from Schneeberg, Saxony, containing an undetermined but small amount of $P$ in substitution for As (Frondel, 1951a). Probably a complete series by mutual substitution of $P$ and As extends. between novacekite and saléeite, and at least a partial series by mutual substitution of $\mathrm{Mg}$ and $\mathrm{Ca}$ probably extends between novacekite and uranospinite. These variations in composition are accompanied'by variations in the interplanar spacings.

\begin{tabular}{c|c||l|l}
\hline$d(\mathrm{~A})$ & $I$ & $d(\mathrm{~A})$ & $I$ \\
\hline 10.15 & 10 & 2.79 & 1 \\
$8.97 *$ & 2 & 2.52 & 3 \\
6.80 & 4 & 2.26 & 3 \\
5.68 & 2 & 2.15 & 2 \\
5.06 & 8 & 2.01 & 2 \\
4.50 & $1^{\mathrm{d}}$ & 1.939 & $1^{\mathrm{d}}$ \\
3.83 & 1 & 1.787 & 3 \\
3.58 & 9 & 1.698 & $1^{\mathrm{d}}$ \\
3.35 & 5 & 1.596 & 3 \\
3.17 & 1 & 1.552 & 1 \\
3.03 & 2 & 1.508 & 1 \\
2.89 & 1 & & \\
\hline
\end{tabular}

*Line possibly due to presence of another phase or hydrate.
d Diffuse.

PARSONSITE

$$
\mathrm{Pb}_{2}\left(\mathrm{UO}_{2}\right)\left(\mathrm{PO}_{4}\right)_{2}(\mathrm{OH})_{4} \cdot 7 \mathrm{H}_{2} \mathrm{O}
$$

Material from the Ruggles pegmatite, Grafton Center, N. H. The original pattern from this locality of Frondel (1950a) had a heavy background, and some of the innermost lines were obscured.

\begin{tabular}{|c|c|c|c|}
\hline$d(\mathrm{~A})$ & $I$ & $d(\mathrm{~A})$ & $I$ \\
\hline $\begin{array}{r}10.16 \\
6.92 \\
6.03 \\
5.75 \\
5.16 \\
4.87 \\
4.25 \\
3.97 \\
3.44 \\
3.28 \\
3.15 \\
3.02 \\
2.95 \\
2.81 \\
2.73 \\
2.63 \\
2.55 \\
2.45 \\
2.37 \\
2.32\end{array}$ & $\begin{array}{c}2 \\
1 \\
2 \\
2 \\
2 \\
2 \\
1 \\
10 \\
4 \\
4 \\
8 \\
3 \\
3 \\
4 \\
4 \\
1 \\
3 \\
2 \\
1 \\
1 \\
1\end{array}$ & $\begin{array}{l}2.27 \\
2.22 \\
2.13 \\
2.10 \\
1.990 \\
1.961 \\
1.921 \\
1.888 \\
1.852 \\
1.788 \\
1.728 \\
1.661 \\
1.629 \\
1.610 \\
1.578 \\
1.506 \\
1.433 \\
1.402 \\
1.370\end{array}$ & $\begin{array}{r}2 \\
3 \\
6 \\
2 \\
1 \\
1 \\
1 / 2 \\
2 \\
5 \\
4 \\
4 \\
6 \\
1 \\
1 \\
1 \\
1 \\
2 \\
1 \\
2\end{array}$ \\
\hline
\end{tabular}




\section{PELIGOTITE}

Identical with johannite.

\section{PHOSPHURAN YLITE \\ $\mathrm{Ca}\left(\mathrm{UO}_{2}\right)_{4}\left(\mathrm{PO}_{4}\right)_{2}(\mathrm{OH})_{4} \cdot 7 \mathrm{H}_{2} \mathrm{O}$}

Data from Frondel (1950b) on material from the Flat Rock mine, Mitchell County, N. C., and from Hogarth and Nuffield (1954) on material from Urgeiriça, Portugal. See also Bignand, Goñi, and Guillemin (1954). The pattern is very similar to that of renardite, with which mineral phosphuranylite forms at least a partial series by mutual substitution of $\mathrm{Ca}$ and $\mathrm{Pb}$.

\begin{tabular}{|c|c|c|c|c|c|c|}
\hline \multicolumn{4}{|c|}{ North Carolina } & \multicolumn{3}{|c|}{ Portugal } \\
\hline$d(\mathrm{~A})$ & $I$ & $d(\mathrm{~A})$ & $I$ & $d(\mathrm{~A})$ & $I$ & hkl \\
\hline $\begin{array}{r}10.16 \\
7.83 \\
7.20 \\
6.33 \\
5.83 \\
5.37 \\
4.96 \\
4.72 \\
4.33 \\
3.97 \\
3.83 \\
3.44 \\
3.36 \\
3.12 \\
3.07 \\
2.94 \\
2.86 \\
2.71 \\
2.59 \\
2.46 \\
2.43 \\
2.24 \\
2.21 \\
2.16 \\
2.10 \\
2.08 \\
2.04 \\
2.00\end{array}$ & $\begin{array}{r}1 \\
10 \\
1 / 2 \\
1 \\
8 \\
1 / 2 \\
1 \\
2 \\
3 \\
9 \\
1 \\
1 \\
2 \\
6 \\
4 \\
2 \\
6 \\
1 \\
1 \\
1 \\
2 \\
1 / 2 \\
1 \\
1 \\
1 / 2 \\
2 \\
1 \\
1\end{array}$ & $\begin{array}{l}1.895 \\
1.852 \\
1.828 \\
1.771 \\
1.719 \\
1.672 \\
1.656 \\
1.585 \\
1.537 \\
1.506 \\
1.433 \\
1.375 \\
1.358 \\
1.304 \\
1.282 \\
1.252 \\
1.236 \\
1.209 \\
1.197 \\
1.179\end{array}$ & $\begin{array}{c}5 \\
1 \\
1 \\
1 \\
3 \\
1 / 2 \\
1 \\
1 \\
2 \\
1 / 2 \\
1 \\
1 \\
1 / 2 \\
1 \\
1 \\
1 \\
1 / 2 \\
1 / 2 \\
1 / 2 \\
1\end{array}$ & $\begin{array}{r}\text { 10. } 34 \\
7.91 \\
5.83 \\
4.92 \\
4.73 \\
4.42 \\
4.30 \\
\text { 3. } 96 \\
3.88 \\
3.81 \\
3.44 \\
\text { 3. } 37 \\
3.15 \\
\\
\text { 3. } 10 \\
\\
\text { 2. } 93 \\
\text { 2. } 88\end{array}$ & $\begin{array}{r}3 \\
10 \\
5 \\
1 / 2 \\
2 \\
3 \\
1 \\
6 \\
3 \\
3 \\
2 \\
1 \\
6\end{array}$ & $\left\{\begin{array}{l}101 \\
200 \\
220 \\
301 \\
212 \\
311 \\
032 \\
103 \\
321 \\
400 \\
232 \\
240 \\
004 \\
402 \\
014 \\
412 \\
204 \\
052 \\
214 \\
143 \\
501 \\
440 \\
060 \\
252 \\
351\end{array}\right.$ \\
\hline
\end{tabular}

PILBARITE

$\mathrm{UO}_{3} \cdot \mathrm{PbO} \cdot \mathrm{ThO}_{2} \cdot 2 \mathrm{SiO}_{2} \cdot 4 \mathrm{H}_{2} \mathrm{O}($ ? $)$

Authentic X-ray powder spacing data are lacking. 


\section{RABBITTITE}

$$
\mathrm{Ca}_{3} \mathrm{Mg}_{3}\left(\mathrm{UO}_{2}\right)_{2}\left(\mathrm{CO}_{3}\right)_{6}(\mathrm{OH})_{4} \cdot 18 \mathrm{H}_{2} \mathrm{O}
$$

Data of Thompson, Weeks, and Sherwood (1955) on material from Emery County, Utah. Data obtained from diffractometer chart; on film, the two inner lines may not register and the lines at 8.24 and 7.79 may overlap.

\begin{tabular}{l|r||l|l}
\hline$d(\mathrm{~A})$ & $I$ & $d(\mathrm{~A})$ & $I$ \\
\hline 19.4 & 3 & 5.22 & 3 \\
18.6 & 1 & 4.81 & 5 \\
11.28 & 5 & 4.71 & 7 \\
8.63 & 3 & 4.51 & 1 \\
8.24 & 10 & 4.37 & 8 \\
7.79 & 8 & 4.28 & 1 \\
7.15 & 3 & 4.05 & 3 \\
6.81 & 1 & 4.03 & 3 \\
6.47 & 3 & 3.84 & 3 \\
6.35 & 1 & $3.71 \mathrm{~b}$ & 1 \\
$5.83 \mathrm{~b}$ & 5 & 3.60 & 3 \\
5.72 & 5 & 3.33 & 5 \\
\hline
\end{tabular}

b Broad.

\section{RAUVITE}

$$
\mathrm{CaO} \cdot 2 \mathrm{UO}_{3} \cdot 2 \mathrm{~V}_{2} \mathrm{O}_{5} \cdot 16 \mathrm{H}_{2} \mathrm{O}(\text { ?) }
$$

'Material from Temple Mountain, San Rafael Swell,'Utah.

\begin{tabular}{r|r||l|l}
\hline$d(\mathrm{~A})$ & \multicolumn{1}{|c||}{$I$} & $d(\mathrm{~A})$ & $I$ \\
\hline 10.70 & 10 & 2.10 & 1 \\
5.83 & 1 & 1.979 & 1 \\
3.87 & 2 & 1.832 & 1 \\
3.49 & 4 & 1.714 & 1 \\
2.95 & 5 & 1.624 & 1 \\
2.62 & 3 & 1.543 & 1 \\
2.22 & 1 & & \\
\hline
\end{tabular}




\section{RENARDITE \\ $\mathrm{Pb}\left(\mathrm{UO}_{2}\right)_{4}\left(\mathrm{PO}_{4}\right)_{2}(\mathrm{OH})_{4} \cdot 7 \mathrm{H}_{2} \mathrm{O}$}

Material from the Katanga district, Belgian Congo. The pattern is very similar to that of phosphuranylite; see also dewindtite.

\begin{tabular}{|c|c|c|c|}
\hline$d(\mathrm{~A})$ & $I$ & $d(\mathrm{~A})$ & $I$ \\
\hline $\begin{array}{l}10.5 \\
7.97 \\
6.37 \\
5.83 \\
4.98 \\
4.80 \\
4.37 \\
3.99 \\
3.82 \\
3.38 \\
3.13 \\
3.09 \\
2.70 \\
2.59 \\
2.54 \\
2.45 \\
2.27 \\
2.22 \\
2.17 \\
2.11 \\
2.09 \\
2.05\end{array}$ & $\begin{array}{r}4 \\
10 \\
3 \\
8 \\
4 \\
4 \\
7 \\
9 \\
5 \\
7 \\
8 \\
8 \\
1{ }^{b} \\
2 \\
2 \\
3 \\
3 \\
3 \\
3 \\
1{ }^{b} \\
4 \\
4\end{array}$ & $\begin{array}{l}2.01 \\
1.95 \\
1.90 \\
1.85 \\
1.82 \\
1.78 \\
1.72 \\
1.70 \\
1.68 \\
1.66 \\
1.60 \\
1.55 \\
1.514 \\
1.497 \\
1.471 \\
1.439 \\
1.377 \\
1.356 \\
1.302 \\
1.290 \\
1.266\end{array}$ & 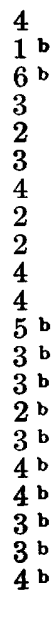 \\
\hline
\end{tabular}

b Broad.

\section{RICHETITE}

A hydrated oxide of uranium and lead. Authentic X-ray powder data for richetite are lacking.

\section{RUTHERFORDINE}

$$
\left(\mathrm{UO}_{2}\right) \mathrm{CO}_{3}
$$

Data from Frondel and Meyrowitz (1956) on synthetic material. The pattern is identical with that of natural material from the known localities.

\begin{tabular}{l|r||l|l}
\hline$d(\mathrm{~A})$ & $I$ & $d(\mathrm{~A})$ & $I$ \\
\hline & & & \\
\hline 4.60 & 10 & 1.874 & 2 \\
4.29 & 8 & 1.734 & 3 \\
3.90 & 6 & 1.658 & 1 \\
3.21 & 9 & 1.603 & 1 \\
2.64 & 4 & 1.588 & 1 \\
2.51 & 1 & 1.510 & 2 \\
2.41 & 2 & 1.435 & 2 \\
2.32 & 3 & 1.388 & 3 \\
2.15 & 4 & 1.373 & 1 \\
2.05 & 5 & 1.346 & 1 \\
1.95 & 1 & 1.318 & 1 \\
1.914 & 2 & 1.275 & 1 \\
\hline
\end{tabular}




\section{SABUGALITE}

\section{$\mathrm{HAl}\left(\mathrm{UO}_{2}\right)_{4}\left(\mathrm{PO}_{4}\right)_{4} \cdot 16 \mathrm{H}_{2} \mathrm{O}$}

Data from Frondel (1951b) on material from Sabugal, Portugal. The pattern is very similar to those of novacekite and saléeite.

\begin{tabular}{l|r||l|l}
\hline$d(\mathrm{~A})$ & $I$ & $d(\mathrm{~A})$ & $I$ \\
\cline { 1 - 2 } 9.69 & 10 & 2.82 & 1 \\
6.56 & 1 & 2.45 & 2 \\
5.59 & 1 & 2.39 & 2 \\
4.86 & 9 & 2.19 & 6 \\
4.39 & 4 & 1.792 & 1 \\
3.47 & 8 & 1.726 & 1 \\
3.36 & 1 & 1.641 & $1 / 2$ \\
3.22 & $1 / 2$ & 1.552 & 1 \\
3.06 & $1 / 2$ & 1.364 & 1 \\
\hline
\end{tabular}

\section{SALÉEITE}

$$
\mathrm{Mg}\left(\mathrm{UO}_{2}\right)_{2}\left(\mathrm{PO}_{4}\right)_{2} \cdot 8-10 \mathrm{H}_{2} \mathrm{O}
$$

Data from Frondel (1951a) on material from Sabugal, Portugal. See further under novacekite.

\begin{tabular}{l|r||l|l}
\hline$d(\mathrm{~A})$ & $I$ & $d(\mathrm{~A})$ & $I$ \\
\hline 9.85 & 10 & 2.39 & 5 \\
8.60 & 3 & 2.30 & 2 \\
6.50 & 2 & 2.25 & 1 \\
5.46 & 3 & 2.19 & 7 \\
4.95 & 8 & 2.14 & 1 \\
4.51 & 4 & 2.10 & 1 \\
4.19 & 2 & 2.04 & 1 \\
3.64 & 1 & 1.919 & 2 rd \\
3.49 & 9 & 1.860 & 1 \\
3.37 & 3 & 1.798 & 3 \\
3.23 & 5 & 1.731 & 3 \\
3.09 & 2 & 1.599 & 2 \\
2.95 & 5 & 1.554 & 2 \\
2.84 & 1 & 1.526 & 1 \\
2.65 & 1 bd & 1.422 & 1 d \\
2.45 & 6 & 1.371 & 2 b \\
\hline b Broad. & d Diffuse. vd Very diffuse. bd Broad, diffuse.
\end{tabular}




\section{SAMIRESITE}

A variety of betafite high in lead; see further under betafite.

\section{SCHOEPITE \\ $2 \mathrm{UO}_{3} \cdot 5 \mathrm{H}_{2} \mathrm{O}$}

Analyzed specimen from the Katanga district, Belgian Congo.

\begin{tabular}{l|r||l|l}
\hline$d(\mathrm{~A})$ & $I$ & $d(\mathrm{~A})$ & $I$ \\
\hline 7.49 & 10 & 1.766 & 3 \\
6.74 & 1 & 1.681 & 1 \\
6.09 & 2 & 1.648 & 5 \\
4.08 & 2 & 1.604 & 3 \\
3.64 & 8 & 1.561 & 1 \\
3.26 & 9 & 1.523 & 1 \\
3.01 & 1 & 1.481 & 2 \\
2.83 & 2 & 1.457 & 2 \\
2.60 & 7 & 1.393 & 2 \\
2.48 & 3 & 1.373 & 3 \\
2.35 & 2 & 1.347 & 1 \\
2.27 & 1 & 1.298 & 2 \\
2.21 & 1 & 1.231 & 1 \\
2.10 & 4 & 1.211 & 2 \\
2.05 & 6 & 1.199 & 2 \\
1.981 & 1 & 1.170 & 3 \\
1.910 & 2 & 1.141 & 2 \\
1.835 & 4 & & \\
\hline
\end{tabular}


SCHROECKINGERITE

\section{$\mathrm{NaCa}_{3}\left(\mathrm{UO}_{2}\right)\left(\mathrm{CO}_{3}\right)_{3}\left(\mathrm{SO}_{4}\right) \mathrm{F} \cdot 10 \mathrm{H}_{2} \mathrm{O}$}

Data from Ross (1955b) on synthetic material. At about $76^{\circ} \mathrm{C}$ in air or at room temperature over concentrated $\mathrm{H}_{2} \mathrm{SO}_{4}$, schroeckingerite loses $6 \mathrm{H}_{2} \mathrm{O}$ and passes (reversibly) to a hexagonal phise not known in nature (Hurlbut, 1954).

\begin{tabular}{l|r|l||l|l|l}
\hline$d(\mathrm{~A})$ & \multicolumn{1}{|c|}{$I$} & $\mathrm{hkl}$ & $d(\mathrm{~A})$ & $I$ & \multicolumn{1}{c}{$\mathrm{hkl}$} \\
\hline 14.28 & 3 & 001 & & \\
\hline 8.48 & 7 & 020 & 3.303 & 1 & 024,114 \\
7.26 & 10 & 021,111 & 3.13 & 1 & $310,043,223,151$, \\
5.421 & 2 & 022,112 & 2.876 & 7 & $134,204$. \\
4.796 & 8 & 200,003 & 2.767 & 1 & 061,331 \\
4.167 & 2 & $220,113,023$ & 2.706 & 1 & 025,115 \\
4.044 & 1 & $041,221,132$ & 2.393 & 2 & 261,401 \\
3.591 & 1 & $042,222,004$ & 2.304 & 1 & $171,351,262,421$ \\
3.362 & 2 & 203 & & & \\
\hline
\end{tabular}

SENGIERITE

$$
\mathrm{Cu}\left(\mathrm{UO}_{2}\right)\left(\mathrm{VO}_{4}\right)(\mathrm{OH}) \cdot 4-5 \mathrm{H}_{2} \mathrm{O}
$$

Data of Donnay and Donnay (1955) on type material frcm the Katanga district, Belgian Congo.

\begin{tabular}{|c|c|c|c|c|c|}
\hline$d(\mathrm{~A})$ & $I$ & hkl & $d(\mathbf{A})$ & $I$ & likl \\
\hline 9.82 & $(\mathrm{vvg})$ & 001 & 2. 832 & (m) & 311 \\
\hline 5. 75 & $(\mathrm{~m})^{\prime}$ & 111 & 2. 744 & (w) & 113 \\
\hline 5. 02 & $(w)$ & 111 & 2. 550 & (m) & $13 \overline{1}, 023$ \\
\hline 4. 91 & $(\mathrm{vg})$ & 002 & 2. 495 & $(v w)$ & 322,131 \\
\hline 4. 179 & (m) & 201 & 2. 398 & $(w)^{\prime}$ & 213 \\
\hline 4. 037 & (m) & 020 & 2. 345 & (vw) & 214 \\
\hline 3.735 & $(s)$ & 021 & 2. 267 & (vw) & 231 \\
\hline 3. 262 & (w) & 003 & 2. 163 & $(w)^{\prime}$ & 420,322 \\
\hline 3. 197 & (s) & 202 & 2. 124 & (m) & 330 \\
\hline 3. 179 & (s) & $220,22 \overline{1}$ & 2. 087 & (m) & 402,033 \\
\hline 3.144 & (s) & 310,022 & 2. 051 & $(\mathrm{w})$ & $51 \overrightarrow{1}$ \\
\hline 3. 094 & (s) & $11 \frac{3}{3}$ & 2.009 & $(\mathrm{m})$ & $42 \overline{3}$ \\
\hline 2. 970 & (m) & 312 & 1. 971 & (vw) & $41 \overline{4}, 14 \overline{1}$ \\
\hline 2. 901 & (vw) & 221 & 1.951 & (vw) & $11 \overline{5}, 21 \overline{5}$ \\
\hline
\end{tabular}

vvs Very, very strong. 


\section{SHARPITE \\ $\left(\mathrm{UO}_{2}\right) \mathrm{CO}_{3} \cdot \mathrm{H}_{2} \mathrm{O}(?)$}

Authentic X-ray powder data for sharpite are lacking.

SKLODOWSKITE

$$
\mathrm{Mg}\left(\mathrm{UO}_{2}\right)_{2}\left(\mathrm{SiO}_{3}\right)_{2}(\mathrm{OH})_{2} \cdot 5 \mathrm{H}_{2} \mathrm{O}
$$

Material from the Katanga district, Belgium Congo. As with other acicular members of the uranophane group, care should be taken to avoid preferred orientation in the preparation of the mount.

\begin{tabular}{l|r||l|l}
\hline$d(\mathrm{~A})$ & \multicolumn{1}{|c|}{$I$} & $d(\mathrm{~A})$ & $I$ \\
\hline & & & \\
\hline 8.42 & 10 & 2.17 & 2 \\
6.37 & 2 & 2.13 & 3 \\
5.91 & 5 & 2.09 & 3 \\
4.82 & 4 & 1.985 & 2 \\
4.51 & 1 & 1.961 & 2 \\
4.33 & 4 & 1.918 & 2 \\
4.19 & 8 & 1.884 & 1 \\
4.00 & 5 & 1.848 & 2 \\
3.52 & 6 & 1.821 & $1 / 2$ \\
3.27 & 7 & 1.801 & 1 \\
3.00 & 6 & 1.768 & $1 \mathrm{~b}$ \\
2.87 & 5 & 1.740 & $1 \mathrm{~b}$ \\
2.80 & 2 & 1.707 & $1 \mathrm{~b}$ \\
2.74 & 2 & 1.672 & $1 / 2$ \\
2.70 & 1 & 1.647 & $1 \mathrm{~b}$ \\
2.66 & 1 & 1.623 & 2 \\
2.52 & 1 & 1.593 & $1 \mathrm{~b}$ \\
2.34 & 2 & 1.563 & 1 \\
2.27 & $1 / 2$ & 1.540 & 1 \\
2.22 & 2 & 1.512 & 1 \\
2.19 & 2 & 1.490 & 1 \\
\hline
\end{tabular}

b Broad. 


\section{SODDYITE \\ $\left(\mathrm{UO}_{2}\right)_{5}\left(\mathrm{SiO}_{4}\right)_{2}(\mathrm{OH})_{2} \cdot 5 \mathrm{H}_{2} \mathrm{O}$}

Material from the Ruggles pegmatite, Grafton Center, N. H. The mineral may have a fine-fibrous habit and care should be taken to. avoid preferred orientation in the mount. The chemical composition of soddyite apparently does not vary significantly.

\begin{tabular}{l|r||l|l}
\hline$d(\mathrm{~A})$ & $I$ & $d(\mathrm{~A})$ & $I$ \\
\hline & & & \\
\hline 6.14 & 8 & 1.699 & 3 \\
4.71 & 3 & 1.664 & 4 \\
4.48 & 9 & 1.641 & 4 \\
3.73 & 2 & 1.601 & 2 \\
3.32 & 10 & 1.551 & 1 \\
3.22 & 2 & 1.523 & 3 \\
2.95 & 3 & 1.503 & 1 \\
2.78 & 2 & 1.463 & 1 \\
2.69 & 7 & 1.406 & 3 \\
2.47 & 6 & 1.374 & 1 \\
2.39 & 1 & 1.353 & 2 \\
2.32 & 2 & 1.339 & 1 \\
2.26 & 2 & 1.289 & 1 \\
2.18 & 1 & 1.268 & 3 \\
2.09 & 4 & 1.220 & 2 \\
2.04 & 3 & 1.148 & 2 \\
1.975 & 4 & 1.082 & 2 \\
1.908 & 4 & 1.055 & 1 \\
1.855 & 5 & 1.038 & 1 \\
1.823 & 1 & 1.018 & 1 \\
1.772 & 2 & .996 & 1 \\
1.731 & 1 & .940 & 1 \\
\hline
\end{tabular}

A lead uranyl carbonate?

A doubtful mineral for which X-ray powder spacing ${ }_{-7}^{7}$ da are lacking. 


\section{SWARTZITE \\ $\mathrm{CaMg}\left(\mathrm{UO}_{2}\right)\left(\mathrm{CO}_{3}\right)_{3} \cdot 12 \mathrm{H}_{2} \mathrm{O}$}

Data from Axelrod, Grimaldi, Milton, and Murata (1951) on material from the Hillside mine, Yavapai County, Utah.

\begin{tabular}{l|r||l|l}
\hline$d(\mathrm{~A})$ & $I$ & $d(\mathrm{~A})$ & $I$ \\
\hline 8.76 & 10 & 3.11 & \\
7.31 & 9 & 3.04 & 2 \\
6.37 & 5 & 2.91 & 8 \\
5.83 & 1 & 2.82 & 2 \\
5.50 & 10 & 2.61 & 5 \\
5.13 & 1 & 2.28 & 4 \\
4.82 & 8 & 2.23 & 3 \\
4.62 & 2 & 2.19 & 2 \\
4.46 & 2 & 2.10 & 2 \\
4.37 & 2 & 2.06 & 8 \\
3.85 & 2 & 1.817 & 5 \\
3.66 & 7 & 1.707 & 8 \\
3.53 & 5 & 1.375 & 2 \\
3.39 & 1 & 1.268 & 2 \\
3.31 & 1 & 1.217 & 3 \\
3.25 & 1 & .962 & 2 \\
3.19 & 7 & & \\
\hline
\end{tabular}

\section{THORIANITE}

\section{$\mathrm{ThO}_{2}$}

Data on pure synthetic $\mathrm{ThO}_{2}$ with $\mathrm{a}_{0}=5.59 \mathrm{~A}$. The unit cell edge of natural thorianite varies from at least 5.50 to $5.58 \mathrm{~A}$ due to substitution of uranium and other elements for thorium, and the spacings vary accordingly. See further under uraninite.

\begin{tabular}{|c|c|c|c|c|c|}
\hline$d(\mathrm{~A})$ & $I$ & hkl & $d(\mathrm{~A})$ & $I$ & hkl \\
\hline $\begin{array}{l}\text { 3. } 21 \\
\text { 2. } 78 \\
1.971 \\
1.682 \\
1.611 \\
1.396 \\
1.280 \\
1.248 \\
1.141 \\
1.076\end{array}$ & $\begin{array}{r}10 \\
4 \\
8 \\
9 \\
2 \\
2 \\
4 \\
4 \\
4 \\
4\end{array}$ & $\begin{array}{l}111 \\
200 \\
220 \\
311 \\
222 \\
400 \\
331 \\
420 \\
422 \\
511,333\end{array}$ & $\begin{array}{r}0.988 \\
.945 \\
.932 \\
.884 \\
.853 \\
.844 \\
.808 \\
.784 \\
.776\end{array}$ & $\begin{array}{l}3 \\
6 \\
3 \\
4 \\
3 \\
3 \\
2 \\
7 \\
8\end{array}$ & $\begin{array}{l}440 \\
531 \\
660,442 \\
620 \\
533 \\
622 \\
444\end{array}$ \\
\hline
\end{tabular}




\section{THORITE}

\section{$\mathrm{ThSiO}_{4}$}

Data on pure anhydrous synthetic tetragonal $\mathrm{ThSiO}_{4}$. Natural thorite is nearly or completely metamict. The spacings of such material when recrystallized by heating may differ slightly from those of pure synthetic material due to a variable and sometimes large content of U, rare earths, or other elements in substitution for Th.

\begin{tabular}{|c|c|c|c|c|c|}
\hline$d(\mathrm{~A})$ & $I$ & hkl & $d(\mathrm{~A})$ & $I$ & hkl \\
\hline $\begin{array}{l}\text { 4. } 69 \\
3.56 \\
2.84 \\
2.66 \\
2.52 \\
2.22 \\
\text { 2. } 01 \\
1.89 \\
1.84 \\
1.787 \\
1.749 \\
1.672 \\
1.599 \\
1.571 \\
1.486 \\
1.438 \\
1.396 \\
1.336 \\
1.282 \\
1.264 \\
1.182 \\
1.168\end{array}$ & $\begin{array}{r}9 \\
10 \\
7 \\
8 \\
5 \\
1 / 2 \\
5 \\
6 \\
10 \\
5 \\
6 \\
3 \\
5 \\
4 \\
6 \\
7 \\
6 \\
8 \\
7 \\
1 / 2 \\
7 \\
1\end{array}$ & $\begin{array}{l}011 \\
200 \\
121 \\
112 \\
220 \\
022 \\
013 \\
321 \\
312 \\
400 \\
123 \\
411 \\
004 \\
240,033 \\
332 \\
024 \\
431 \\
224 \\
152 \\
440 \\
044,215,600,343,503 \\
611\end{array}$ & $\begin{array}{l}1.145 \\
1.123 \\
1.059 \\
1.049 \\
1.024 \\
1.016 \\
.990 \\
.984 \\
.972 \\
.950 \\
.918 \\
.900 \\
.890 \\
.875 \\
.865 \\
.861 \\
.840 \\
.839 \\
.819 \\
.805 \\
.798\end{array}$ & 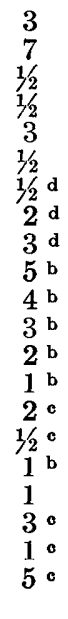 & $\begin{array}{l}532 \\
244,305,260,523 \\
602 \\
325 \\
116,631 \\
415 \\
613,206,701 \\
444,460 \\
543 \\
316,721,552,712 \\
624,525 \\
336 \\
732 \\
800 \\
811,741 \\
615 \\
820 \\
714 \\
156 \\
644 \\
660\end{array}$ \\
\hline
\end{tabular}

d Diffuse line. b Diffuse line, possibly a doublet.

- Broad band, centering as listed. 


\section{THOROGUMMITE}

\section{Ideally $\mathrm{Th}\left(\mathrm{SiO}_{4}\right)_{1-\mathrm{x}}(\mathrm{OH})_{4 \mathrm{x}}$}

Data from Frondel (1953) on material from Baringer Hill, Tex. The pattern, virtually identical with that of thorite, often is rather diffuse due to small particle size. Natural material contains variable amounts of uranium, rare earths, and other cations in substitution for Th and the content of $(\mathrm{OH})$ is variable, but nothing is known of the attendant variation in spacings. Tetragonal.

\begin{tabular}{|c|c|c|c|c|c|}
\hline$d(\mathrm{~A})$ & $I$ & $\mathrm{hkl}$ & $d(\mathbf{A})$ & $I$ & hkl \\
\hline $\begin{array}{l}\text { 4. } 695 \\
\text { 3. } 537 \\
\text { 2. } 821 \\
\text { 2. } 653 \\
\text { 2. } 499 \\
\text { 2. } 336 \\
\text { 2. } 203 \\
\text { 2. } 000 \\
\text { 1. } 869 \\
\text { 1. } 818 \\
\text { 1. } 767 \\
\text { 1. } 740 \\
\text { 1. } 653 \\
\text { 1. } 582 \\
\text { 1. } 565 \\
\text { 1. } 469 \\
\text { 1. } 431\end{array}$ & $\begin{array}{r}9 \\
10 \\
4 \\
6 \\
3 \\
1 / 2 \\
4 \\
4 \\
3 \\
6 \\
1 \\
1 \\
1 \\
1 \\
1 \\
2 \\
3\end{array}$ & $\begin{array}{l}011 \\
200 \\
121 \\
112 \\
220 \\
022 \\
031 \\
013 \\
321 \\
312 \\
400 \\
123 \\
411 \\
420 \\
004 \\
332 \\
024\end{array}$ & $\begin{array}{c}1.379 \\
1.326 \\
1.267 \\
1.172 \\
1.130 \\
1.112 \\
1.088 \\
1.055 \\
1.039 \\
1.021 \text { to } 1.013 \\
.976 \\
.953 \text { to } .951 \\
.908 \\
.888 \\
.833 \\
.807 \\
.791\end{array}$ & 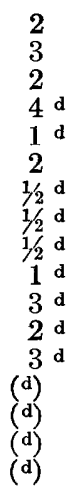 & $\begin{array}{l}431 \\
224 \\
431 \\
044 \\
532 \\
244 \\
541 \\
622 \\
631 \\
415,116 \\
444 \\
552 \\
624 \\
800 \\
644 \\
662 \\
840\end{array}$ \\
\hline
\end{tabular}

d Diffuse. 


\section{TORBERNITE}

$$
\mathrm{Cu}\left(\mathrm{UO}_{2}\right)_{2}\left(\mathrm{PO}_{4}\right)_{2} \cdot 8-12 \mathrm{H}_{2} \mathrm{O}
$$

Data from Berman (1956) on fully hydrated synthetic material. The chemical composition of this species is essentially constant aside from variation in the content of zeolitic water, and patterns of natural material from different localities show few or no significant differences. Torbernite rapidly dehydrates under warm, dry atmospheric conditions to metatorbernite. The pattern of torbernite is relatively close to that of zeunerite.

\begin{tabular}{c|r||l|l}
\hline$d(\mathrm{~A})$ & $I$ & $d(\mathrm{~A})$ & $I$ \\
\hline 10.3 & 10 & 2.67 & 1 \\
6.61 & 4 & 2.48 & 1 \\
5.18 & 3 & 2.41 & 2 \\
4.94 & 9 & 2.37 & 1 \\
4.48 & 4 & 2.32 & 1 \\
3.67 & 4 & 2.24 & 1 \\
3.58 & 9 & 2.21 & 3 \\
3.51 & 8 & 2.158 & 2 \\
3.10 & 1 & 2.040 & 3 \\
2.90 & 3 & 1.917 & 1 \\
2.85 & 2 & 1.873 & 1 \\
2.73 & 1 & & \\
\hline
\end{tabular}




\section{TROEGERITE}

$$
\mathrm{H}_{2}\left(\mathrm{UO}_{2}\right)_{2}\left(\mathrm{AsO}_{4}\right)_{2} \cdot 8 \mathrm{H}_{2} \mathrm{O}(?)
$$

The chemical composition of troegerite is in doubt, and type specimens of the mineral are not available. The existing evidence indicates that troegerite has the composition given above, and the present spacing data from Mrose (1953) have been obtained on synthetic material of this composition.

\begin{tabular}{|c|c|c|c|}
\hline$d(\mathrm{~A})$ & $I$ & $d(\mathrm{~A})$ & $I$ \\
\hline $\begin{array}{l}8.59 \\
5.50 \\
5.04 \\
4.35 \\
3.79 \\
3.56 \\
3.30 \\
3.14 \\
2.99 \\
2.76 \\
2.70 \\
2.59 \\
2.53 \\
2.43 \\
2.30 \\
2.26 \\
2.19 \\
2.16 \\
2.10 \\
2.01 \\
1.931 \\
1.873 \\
1.845 \\
1.807 \\
1.787 \\
1.752 \\
1.705 \\
1.685 \\
1.658 \\
1.643 \\
1.613\end{array}$ & $\begin{array}{r}10 \\
7 \\
4 \\
7 \\
9 \\
6 \\
8 \\
1 / 2 \\
5 \\
2 \\
7 \\
3 \\
6 \\
5 \\
2 \\
3 \\
7 \\
4 \\
5 \\
7 \\
3 \\
2 \\
3 \\
4 \\
4 \\
4 \\
1 \\
1 / 2 \\
7 \\
2 \\
3\end{array}$ & $\begin{array}{l}1.598 \\
1.576 \\
1.541 \\
1.508 \\
1.492 \\
1.473 \\
1.462 \\
1.437 \\
1.427 \\
1.388 \\
1.360 \\
1.335 \\
1.316 \\
1.297 \\
1.285 \\
1.275 \\
1.265 \\
1.252 \\
1.239 \\
1.230 \\
1.217 \\
1.211 \\
1.182 \\
1.169 \\
1.139 \\
1.133 \\
1.122 \\
1.110 \\
1.098\end{array}$ & $\begin{array}{r}3 \\
7 \\
1 / 2 \\
1 / 2 \\
1 \\
1 / 2 \\
1 \\
4 \\
4 \\
6 \\
3 \\
4 \\
1 \\
1 / 2 \\
3 \\
1 / 2 \\
1 / 2 \\
2 \\
2 \\
2 \\
2 \\
1 / 2 \\
5 \\
4 \\
1 / 2 \\
2 \\
4 \\
2 \\
4\end{array}$ \\
\hline
\end{tabular}




\section{$\mathrm{Ca}\left(\mathrm{UO}_{2}\right)_{2}\left(\mathrm{VO}_{4}\right)_{2} \cdot 5-8.5 \mathrm{H}_{2} \mathrm{O}$}

Fully hydrated natural material from Hudspeth County, Tex. The water content varies zeolitically between 5 and 8.5 with attendant variation in the spacings of (001) and other planes; the spacings decrease with decreasing water content. Data for tyuyamunite of controlled water content also are given by Stern and others (1956). Metatyuyamunite is a common impurity. Tyuyamunite, metatyuyamunite, carnotite and, to a less extent, zippeite resemble each other in the hand specimens but their X-ray patterns are distinctive.

\begin{tabular}{r|r||l|l}
\hline$d(\mathrm{~A})$ & $I$ & $d(\mathrm{~A})$ & $I$ \\
\hline 10.18 & 10 & 2.48 & 1 \\
6.62 & 3 & 2.28 & 1 \\
5.02 & 9 & 2.16 & 1 \\
4.02 & 2 & 2.10 & 1 \\
3.37 & 3 & 2.04 & 4 \\
3.20 & 5 & 1.964 & 1 \\
3.12 & 3 & 1.911 & 2 \\
2.69 & 1 & 1.870 & 1 \\
2.55 & 2 & & \\
\hline
\end{tabular}

\section{UMOHOITE}

\section{$\left(\mathrm{UO}_{2} \mathrm{MoO}_{4} \cdot 4 \mathrm{H}_{2} \mathrm{O}(?)\right.$}

Data of Brophy and Kerr (1953) on material from Marysvale, Utah. Relative intensities from spectrometer chart peaks. The pattern varies due to effects of X-ray irradiation, and the present data were obtained on a sample previously irradiated for two days.

\begin{tabular}{r|r||l|r}
\hline$d(\mathrm{~A})$ & \multicolumn{1}{|c||}{$I$} & $d(\mathrm{~A})$ & \multicolumn{1}{|c}{$I$} \\
\hline 14.29 & 52 & 3.06 & 7 \\
8.57 & 23 & 2.891 & 17 \\
7.19 & 264 & 2.402 & 1 \\
4.80 & 176 & 2.149 & 2 \\
4.30 & 3 & 2.061 & 45 \\
3.72 & 2 & 1.807 & 8 \\
3.60 & 62 & 1.606 & 3 \\
3.22 & 10 & 1.443 & 4 \\
\hline
\end{tabular}


$\mathrm{UO}_{2}$

Data on essentially stoichiometric synthetic $\mathrm{UO}_{2}$ with $\mathrm{a}_{0}=5.46 \mathrm{~A}$. The unit cell dimensions of natural uraninite vary from at least $5.36 \mathrm{~A}$ to $5.50 \mathrm{~A}$ due to oxidation and the entrance of other elements in substitution for uranium, and the spacings vary accordingly. The pattern is distinctive, and there is little chance of confusion with other rninerals with the exception of thorianite, cerianite, sphalerite, and bindheimite. Thorianite is isostructural with uraninite and patterns are analogous, but the unit cell edge of thorianite is larger and the spacing correspondingly smaller than with uraninite. A complete series exists between $\mathrm{ThO}_{2}$ and $\mathrm{UO}_{2}$, and the patterns of the two minerals are therefore gradational. Cerianite also has an analogous pattern but the cell dimensions of pure $\mathrm{CeO}_{2}$ are smaller than those of all but relatively highly oxidized uraninite. Confirmatory chemical tests are desirable with these minerals.

\begin{tabular}{|c|c|c|c|c|}
\hline \multicolumn{3}{|c|}{$\begin{array}{c}\text { Synthetic } \mathrm{UO}_{2} \\
\mathrm{a}_{0}=5.46 \mathrm{~A}\end{array}$} & \multicolumn{2}{|c|}{$\begin{array}{c}\text { Synthetic } \mathrm{CeO}_{2} * \\
\mathrm{a}_{0}=5.41 \mathrm{~A}\end{array}$} \\
\hline$d(\mathrm{~A})$ & $I$ & hkl & $d(\mathrm{~A})$ & $I$ \\
\hline $\begin{array}{l}3.14 \\
2.73 \\
1.926 \\
1.645 \\
1.574 \\
1.365 \\
1.251 \\
1.220 \\
1.115 \\
1.051 \\
.966 \\
.924 \\
.911 \\
.864 \\
.833 \alpha_{1} \\
.824 \alpha_{1} \\
.789 \alpha_{1}\end{array}$ & $\begin{array}{r}10 \\
5 \\
8 \\
9 \\
4 \\
3 \\
6 \\
6 \\
6 \\
7 \\
4 \\
7 \\
5 \\
6 \\
5 \\
5 \\
3\end{array}$ & $\begin{array}{l}111 \\
002 \\
022 \\
113 \\
222 \\
004 \\
133 \\
024 \\
224 \\
333,115 \\
044 \\
135 \\
006,244 \\
026 \\
335 \\
226 \\
444\end{array}$ & $\begin{array}{r}3.11 \\
2.69 \\
1.90 \\
1.62 \\
1.55 \\
1.35 \\
1.24 \\
1.21 \\
1.10 \\
1.04 \\
.95 \\
.91 \\
.90 \\
.85 \\
.82 \\
.76 \\
.72 \\
.70\end{array}$ & $\begin{array}{r}10 \\
3 \\
8 \\
6 \\
1 \\
1 \\
3 \\
2 \\
2 \\
2 \\
1 / 2 \\
1 \\
1 / 2 \\
1 / 2 \\
1 / 2 \\
1 / 2 \\
1 / 2 \\
1 / 2\end{array}$ \\
\hline
\end{tabular}

*Data from ASTM card file. 


\section{URANOPHANE \\ $\mathrm{Ca}\left(\mathrm{UO}_{2}\right)_{2}\left(\mathrm{SiO}_{3}\right)_{2}(\mathrm{OH})_{2} \cdot 5 \mathrm{H}_{2} \mathrm{O}$}

Material from the Ruggles pegmatite, Grafton Center, N. H. Direct comparison of films taken of material from numerous localities shows little or no significant variations in spacing. The dense types of uranophane give good patterns with conventional methods of mount preparation, but care should be taken with acicular types to avoid preferred orientation. The patterns of uranophane and its polymorph beta-uranophane are distinctly different although the two minerals are very similar in the hand specimen.

\begin{tabular}{l|r||l|l}
\hline$d(\mathrm{~A})$ & $I$ & $d(\mathrm{~A})$ & $I$ \\
\hline 7.88 & 10 & & \\
\hline 6.61 & 4 & 2.06 & \\
5.42 & 4 & 1.969 & 7 \\
4.76 & 5 & 1.906 & 2 \\
4.29 & 2 & 1.869 & 1 \\
3.94 & 9 & 1.827 & 3 \\
3.60 & 4 & 1.769 & 1 \\
3.51 & 4 & 1.747 & 3 \\
3.41 & 1 & 1.717 & 3 \\
3.35 & 1 & 1.659 & 1 \\
3.20 & 5 & 1.601 & 1 \\
3.09 & 1 & 1.579 & 2 \\
2.99 & 8 & 1.537 & 2 \\
2.91 & 8 & 1.496 & 2 \\
2.69 & 4 & 1.461 & 2 \\
2.63 & 5 & 1.442 & 2 \\
2.57 & 2 & 1.382 & 1 \\
2.52 & 2 & 1.372 & 1 \\
2.40 & 1 & 1.345 & 1 \\
2.26 & 2 & 1.325 & 1 \\
2.20 & 4 & 1.262 & 1 \\
2.10 & 5 & 1.239 & 2 \\
\hline
\end{tabular}




\section{URANOPILITE}

$$
\left(\mathrm{UO}_{2}\right)_{6}\left(\mathrm{SO}_{4}\right)(\mathrm{OH})_{10} \cdot 12 \mathrm{H}_{2} \mathrm{O}
$$

Data from Frondel (1952) on material from Wheal Owles, Cornwall, England. Identical patterns are afforded by material from other localities. Gypsum is characteristically admixed or associated with uranopilite and zippeite and may give rise to extra lines in the pattern. The fine-fibrous character of uranopilite may cause preferred orientation in the mount.

\begin{tabular}{l|r||l|l}
\hline$d(\mathrm{~A})$ & $I$ & $d(\mathrm{~A})$ & $I$ \\
\hline & & & \\
\cline { 1 - 2 } 9.18 & 8 & 2.42 & 1 \\
8.21 & 2 & 2.36 & 1 \\
7.12 & 10 & 2.28 & 1 \\
5.99 & 2 & 2.24 & 1 \\
5.51 & 4 & 2.19 & 3 \\
5.13 & 1 & 2.13 & 1 \\
4.76 & 1 & 2.08 & 1 \\
4.61 & 1 & 2.04 & 1 \\
4.28 & 8 & 2.01 & 1 \\
4.02 & 1 & 1.932 & 2 \\
3.86 & 2 & 1.880 & 2 \\
3.65 & 5 & 1.826 & 3 \\
3.48 & 2 & 1.757 & 3 \\
3.31 & 4 & 1.732 & 1 \\
3.08 & 1 & 1.700 & 2 \\
2.99 & 3 & 1.674 & 1 \\
2.90 & 3 & 1.638 & 1 \\
2.75 & 1 & 1.604 & 1 \\
2.69 & 2 & & \\
2.59 & 2 & & \\
\hline
\end{tabular}




\section{URANOSPATHITE}

The pattern of uranospathite is virtually identical with that of fully hydrated zeunerite but with extra limes at $d 14.6$ and $d 7.56$. (See Frondel, 1954.) The fresh mineral dehydrates very rapidly and then gives the pattern of metazeunerite. The status of uranospathite is uncertain. The mineral appears to be a copper uranyl phosphatearsenate related to the members of the torbernite group but containing: a larger amount of $\mathrm{H}_{2} \mathrm{O}$.

\section{URANOSPHERITE}

$(\mathrm{BiO})\left(\mathrm{UO}_{2}\right)(\mathrm{OH})_{3}(?)$

Material from Schneeberg, Saxony.

\begin{tabular}{l|r||l|l}
\hline$d(\mathrm{~A})$ & $I$ & $d(\mathrm{~A})$ & $I$ \\
\hline & & & \\
\hline 5.25 & 6 & 1.90 & 5 \\
4.74 & 3 & 1.83 & 8 \\
4.37 & 4 & 1.80 & 1 \\
4.15 & 1 & 1.73 & 2 \\
3.87 & 7 & 1.66 & 4 \\
3.47 & 6 & 1.59 & 1 \\
3.16 & 10 & 1.56 & 1 \\
3.05 & 3 & 1.52 & 1 \\
2.71 & 1 & 1.37 & 1 \\
2.60 & 1 & 1.34 & 1 \\
2.54 & 1 & 1.33 & 1 \\
2.46 & 2 & 1.26 & 2 \\
2.39 & 2 & 1.236 & 2 \\
2.35 & 1 & 1.198 & 1 \\
2.16 & 2 & 1.181 & 4 \\
2.08 & 2 & 1.097 & 1 \\
2.03 & 1 & 1.058 & 1 \\
1.97 & 4 & & \\
\hline
\end{tabular}


X-RAY POWDER DATA FOR URANIUM AND THORIUM MINERALS 145

URANOSPINITE

$\mathrm{Ca}\left(\mathrm{UO}_{2}\right)_{2}\left(\mathrm{AsO}_{4}\right)_{2} \cdot 8-10 \mathrm{H}_{2} \mathrm{O}$

Data from Mrose (1953) on synthetic material containing $8 \mathrm{H}_{2} \mathrm{O}$. Data also are given for synthetic material with $\sim 10 \mathrm{H}_{2} \mathrm{O}$.

\begin{tabular}{|c|c|c|c|}
\hline \multicolumn{2}{|c|}{$8 \mathrm{H}_{2} \mathrm{O}$} & \multicolumn{2}{|c|}{$10 \mathrm{H}_{2} \mathrm{O}$} \\
\hline$d(\mathrm{~A})$ & $I$ & $d(\mathbf{A})$ & $I$ \\
\hline $\begin{array}{l}8.85 \\
5.57 \\
5.10 \\
\text { 4. } 40 \\
3.74 \\
3.59 \\
3.34 \\
3.02 \\
2.81 \\
\text { 2. } 68 \\
2.55 \\
2.44 \\
2.29 \\
2.20 \\
2.16 \\
2.10 \\
2.00 \\
1.945 \\
1.845 \\
1.804 \\
1.755 \\
1.704 \\
1.642 \\
1.608 \\
1.575 \\
1.499 \\
1.414 \\
1.384 \\
1.361\end{array}$ & $\begin{array}{r}10 \\
6 \\
7 \\
2 \\
5 \\
9 \\
8 \\
4 \\
1 \\
1 \\
5 \\
4 \\
3 \\
2 \\
2 \\
2 \\
1 \\
1 \\
1 / 2 \\
5 \\
1 \\
1 \\
3 \\
3 \\
3 \\
1 / 2 \\
1 / 2 \\
1 / 2 \\
1 / 2\end{array}$ & $\begin{array}{r}10.16 \\
6.71 \\
5.13 \\
4.90 \\
4.53 \\
3.56 \\
3.39 \\
3.16 \\
2.91 \\
2.82 \\
\text { 2. } 70 \\
2.52 \\
2.46 \\
2.37 \\
2.27 \\
2.21 \\
2.16 \\
2.05 \\
1.90 \\
1.848 \\
1.791 \\
1.765 \\
1.689 \\
1.645 \\
1.618 \\
1.600 \\
1.580 \\
1.535 \\
1.514 \\
1.492 \\
1.404 \\
1.361 \\
1.339 \\
1.302 \\
1.285 \\
1.258 \\
1.229 \\
1.185 \\
1.159 \\
1.127\end{array}$ & 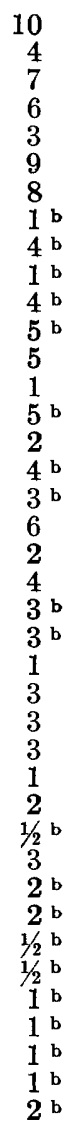 \\
\hline
\end{tabular}

- Broad. 


\section{UVANITE \\ $\mathrm{U}_{2} \mathrm{~V}_{6} \mathrm{O}_{21} \cdot 15 \mathrm{H}_{2} \mathrm{O}(?)$}

Data obtained from five different sources, all derived from type material from Temple Mountain, San Rafael Swell, Utah, are not wholly consistent, probably due to the presence of impurities and to $\checkmark$ ariation in film intensity and quality.

\begin{tabular}{|c|c|c|c|c|c|c|c|c|c|}
\hline \multicolumn{2}{|c|}{$\begin{array}{c}\text { Harvard } \\
\text { standard file }\end{array}$} & \multirow{2}{*}{$d(\mathrm{~A})$} & \multirow{2}{*}{$I$} & \multirow{2}{*}{$d(\mathbf{A})$} & \multirow{2}{*}{$I$} & \multirow{2}{*}{$d(\mathrm{~A})$} & \multirow{2}{*}{$I$} & \multirow{2}{*}{$d(\mathbf{A})$} & \multirow{2}{*}{$I$} \\
\hline$d(\mathbf{A})$ & $I$ & & & & & & & & \\
\hline $\begin{array}{l}5.9 \\
4.6 \\
3.3 \\
2.94 \\
2.24 \\
1.71 \\
1.637 \\
1.484 \\
1.356 \\
1.288 \\
1.180 \\
1.119\end{array}$ & $\begin{array}{r}6 \\
4 \\
2 \\
10 \\
8 \\
9 \\
4 \\
7 \\
2 \\
3 \\
1 \\
4\end{array}$ & $\begin{array}{l}5.9 \\
5.3 \\
3.39 \\
\text { 2. } 96 \\
2.24 \\
1.71 \\
1.64 \\
1.49\end{array}$ & $\begin{array}{c}5 \mathrm{~b} \\
4^{\mathrm{b}} \\
2 \\
10 \\
5^{\mathrm{b}} \\
8 \\
1^{\mathrm{b}} \\
4\end{array}$ & $\begin{array}{l}9.56 \\
5.72 \\
4.55 \\
\\
2.93 \\
2.202 \\
1.695\end{array}$ & $\begin{array}{l}\text { (w) } \\
\text { (w) } \\
\text { (vw) } \\
\text { (vs) } \\
\text { (w) } \\
\text { (w) }\end{array}$ & $\begin{array}{l}\text { 5. } 79 \\
\text { 4. } 60 \\
\text { 3. } 34 \\
\text { 2. } 94 \\
\text { 2. } 21 \\
\text { 1. } 701 \\
\text { 1. } 626 \\
\text { 1. } 475\end{array}$ & $\begin{array}{r}6 \\
7 \\
2 \\
10 \\
4 \\
5 \\
1 \\
3\end{array}$ & $\begin{array}{l}\text { 3. } 36 \\
\text { 2. } 948 \\
\text { 2. } 217 \\
\text { 1. } 698 \\
\\
\text { 1. } 468\end{array}$ & $\begin{array}{l}\text { (w) } \\
\text { (vs) } \\
\text { (s) } \\
\text { (w) } \\
\text { (w) }\end{array}$ \\
\hline
\end{tabular}

- Strong. ve Very strong. W Weak. v" Very weak. b Broad. 


\section{VANDENBRANDEITE}

$$
\mathrm{Cu}\left(\mathrm{UO}_{2}\right) \mathrm{O}_{2} \cdot 2 \mathrm{H}_{2} \mathrm{O}
$$

Data from Milne and Nuffield (1951) on material from Kalongwe, Katanga, Belgian Congo, and from Harvard file on Katanga material. The two sets of data are slightly divergent for reasons not known. See also Bignand (1955).

\begin{tabular}{|c|c|c|c|c|c|}
\hline \multicolumn{4}{|c|}{ Milne and Nuffield, 1951* } & \multirow{2}{*}{$d(\mathrm{~A})$} & \multirow{2}{*}{$I$} \\
\hline$d(\mathrm{~A})$ & $I$ & $d(\mathbf{A})$ & $I$ & & \\
\hline 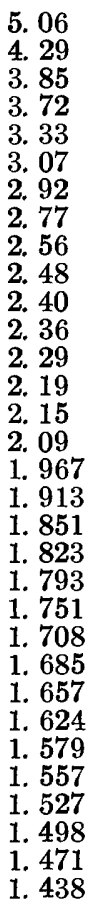 & $\begin{array}{r}4 \\
10 \\
\mathbf{2} \\
\mathbf{2} \\
\mathbf{2} \\
1 \\
8 \\
1 / 2 \\
4 \\
1 / 2 \\
1 / 2 \\
1 \\
1 \\
1 / 2 \\
1 / 2 \\
3 \\
2 \\
1 \\
3 \\
1 / 2 \\
1 \\
1 / 2 \\
1 \\
1 / 2 \\
1 / 2 \\
2 \\
1 / 2 \\
1 \\
1 \\
1 \\
3 \\
1 / 2\end{array}$ & $\begin{array}{l}1.420 \\
1.398 \\
1.348 \\
1.333 \\
1.317 \\
1.300 \\
1.276 \\
1.253 \\
1.246 \\
1.227 \\
1.197 \\
1.173 \\
1.156 \\
1.129 \\
1.096 \\
1.076 \\
1.068 \\
1.055 \\
1.044 \\
1.038 \\
1.028 \\
1.020 \\
1.012 \\
1.003 \\
.990 \\
.978 \\
.968 \\
.954 \\
.944 \\
.939 \\
.931 \\
.927\end{array}$ & $\begin{array}{c}1 / 2 \\
1 / 2 \\
2 \\
1 / 2 \\
3 \\
1 / 2 \\
1 / 2 \\
2 \\
1 / 2 \\
1 \\
1 / 2 \\
1 / 2 \\
1 / 2 \\
1 / 2 \\
2 \\
2 \\
112 \\
1 / 2 \\
1 / 2 \\
1 / 2 \\
1 / 2 \\
1 / 2 \\
1 / 2 \\
1 / 2 \\
1 \\
1 / 2 \\
1 / 2 \\
1 \\
1 \\
1 \\
1 \\
1 / 2\end{array}$ & 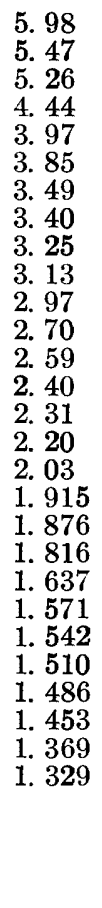 & $\begin{array}{r}3 \\
3 \\
9 \\
10 \\
3 \\
4 \\
1 \\
4 \\
2 \\
2 \\
8 \\
2 \\
7 \\
2 \\
2 \\
5 \\
6 \\
1 \\
3 \\
1 \\
5 \\
1 \\
1 \\
1 \\
2 \\
1 \\
2 \\
2\end{array}$ \\
\hline
\end{tabular}

*Data, in $\mathrm{kx}$ units, converted to angstrom units. 
$\mathrm{PbO} \cdot 7 \mathrm{UO}_{3} \cdot 12 \mathrm{H}_{2} \mathrm{O}$

Material from the Katanga district, Belgian Congo. Optical and other evidence indicate that the two sets of slightly divergent data here reported both represent essentially the same mineral. See further under fourmarierite.

\begin{tabular}{l|l||l|r}
\hline$d(\mathrm{~A})$ & $I$ & $d(\mathrm{~A})$ & $I$ \\
\hline 7.41 & & & \\
\hline 6.49 & 8 & 8.27 & 2 \\
4.58 & 2 & 7.31 & 10 \\
4.27 & $2 \mathrm{~d}$ & 6.49 & 3 \\
3.94 & 1 & 4.85 & 1 \\
3.61 & 1 & 4.39 & 2 \\
3.22 & 9 & 4.14 & 1 \\
2.83 & 10 & 3.93 & 2 \\
2.55 & 2 & 3.58 & 8 \\
2.43 & 4 & 3.37 & 1 \\
2.32 & 3 & 3.19 & 9 \\
2.05 & 2 & 3.00 & 1 \\
2.01 & 3 & 2.73 & 3 \\
1.886 & 4 & 2.53 & 5 \\
1.811 & $1 \mathrm{~d}$ & 2.40 & 2 \\
1.783 & 3 & 2.32 & 1 \\
1.734 & 3 & 2.05 & 2 \\
1.687 & 2 & 1.989 & 7 \\
1.646 & 1 & 1.908 & 3 \\
1.613 & 1 & 1.797 & 3 \\
1.563 & 2 & 1.773 & 5 \\
1.524 & 1 & 1.726 & 3 \\
1.420 & 2 & 1.699 & 2 \\
1.381 & 1 d & 1.657 & 3 \\
1.338 & $1 \mathrm{~d}$ & 1.601 & 4 \\
1.304 & 2 & 1.566 & 2 \\
1.266 & 1 & 1.510 & 2 \\
1.202 & 2 & 1.419 & 2 \\
& 1 & 1.349 & 2 \\
& & 1.332 & 2 \\
& & 1.312 & 1 \\
& & 1.263 & 2 \\
\hline
\end{tabular}

- Diffuse. 
A calcium copper uranyl carbonate

Authentic X-ray powder data are lacking.

WALPURGITE

$$
\mathrm{Bi}_{4}\left(\mathrm{UO}_{2}\right)\left(\mathrm{AsO}_{4}\right)_{2} \cdot 3 \mathrm{H}_{2} \mathrm{O}
$$

Material from Schneeberg, Saxony, Germany. Strong patterns are difficult to obtain due to the low symmetry and highly absorbing nature of the mineral. The spacings vary with substitution of $\mathrm{P}$ for As.

\begin{tabular}{|c|c|c|c|}
\hline$d(\mathbf{A})$ & $I$ & $d(\mathrm{~A})$ & $I$ \\
\hline $\begin{array}{c}10.65 \\
9.9 \\
6.56 \\
5.64 \\
\text { 5. } 44 \\
\text { 5. } 01 \\
4.87 \\
4.10 \\
3.97 \\
3.44 \\
\text { 3. } 38 \\
3.25 \\
3.11 \\
\text { 3. } 05 \\
2.72 \\
\text { 2. } 59\end{array}$ & $\begin{array}{c}1 \\
4 \\
1 \\
1 \\
1 \\
1 / 2 \\
1 \\
1 / 2 \\
1 \\
2 \\
2 \\
5 \\
10 \\
5 \\
4 \\
1{ }^{b}\end{array}$ & $\begin{array}{l}2.50 \\
2.41 \\
2.33 \\
2.24 \\
2.19 \\
2.12 \\
2.07 \\
2.002 \\
1.922 \\
1.895 \\
1.852 \\
1.807 \\
1.774 \\
1.695 \\
1.695\end{array}$ & $\begin{array}{l}1 \text { b } \\
4 \text { b } \\
11 \mathrm{~b} \\
1{ }^{\mathrm{b}} \\
3 \\
2 \\
2 \mathrm{~b} \\
2 \mathrm{~b} \\
3 \mathrm{~b} \\
2 \mathrm{~b} \\
3 \mathrm{~b} \\
3 \mathrm{~b} \\
3 \mathrm{~b} \\
3 \mathrm{~b} \\
4 \\
4 \\
4\end{array}$ \\
\hline
\end{tabular}

b Broad. 


\section{ZEUNERITE \\ $\mathrm{Cu}\left(\mathrm{UO}_{2}\right)_{2}\left(\mathrm{AsO}_{4}\right)_{2} \cdot 8-10 \mathrm{H}_{2} \mathrm{O}$}

Data from J. W. Frondel (1951) on synthetic material containing $16 \mathrm{H}_{2} \mathrm{O}$ and $10 \mathrm{H}_{2} \mathrm{O}$.

\begin{tabular}{|c|c|c|c|c|c|c|c|}
\hline \multicolumn{4}{|c|}{$10 \mathrm{H}_{2} \mathrm{O}$} & \multicolumn{4}{|c|}{$16 \mathrm{H}_{2} \mathrm{O}$} \\
\hline$d(\mathrm{~A})$ & $I$ & $d(\mathrm{~A})$ & $I$ & $d(\mathrm{~A})$ & $I$ & $d(\mathrm{~A})$ & $I$ \\
\hline $\begin{array}{c}10.33 \\
9.40 \\
6.82 \\
5.27 \\
5.07 \\
4.58 \\
3.60 \\
3.40 \\
3.18 \\
2.94 \\
2.86 \\
2.75 \\
2.54 \\
2.47 \\
2.32 \\
2.27 \\
2.19 \\
2.07 \\
1.926 \\
1.869 \\
1.798 \\
1.772 \\
1.707 \\
1.692 \\
1.660 \\
1.642 \\
1.605\end{array}$ & $\begin{array}{c}9 \\
1 \\
4 \\
5 \\
7 \\
2 \\
2 \\
10 \\
6 \\
2 \\
4 \\
1 \\
2 \\
3 \\
3 \\
1 \\
3 \\
3 \\
4 \\
4 \\
1 \\
4 \\
1 \\
2 \\
2 \\
2 \\
2 \\
3\end{array}$ & $\begin{array}{l}1.583 \\
1.561 \\
1.536 \\
1.506 \\
1.428 \\
1.404 \\
1.378 \\
1.358 \\
1.313 \\
1.293 \\
1.270 \\
1.242 \\
1.217 \\
1.164 \\
1.138 \\
1.093 \\
1.083 \\
1.056 \\
1.036 \\
1.022 \\
.997 \\
.972 \\
.959 \\
.947 \\
.929\end{array}$ & $\begin{array}{l}1 \\
1 \\
3 \\
1 \\
2 \\
1 \\
2 \\
2 \\
1 \\
1 \\
1 \\
1 \\
1 \\
1 \\
1 \\
1 \\
1 \\
1 \\
1 \\
1 \\
1 \\
1 \\
1 \\
1 \\
1 \\
1 \\
1\end{array}$ & $\begin{array}{r}10.65 \\
6.86 \\
5.04 \\
4.55 \\
3.59 \\
3.39 \\
3.18 \\
3.07 \\
2.93 \\
2.86 \\
2.73 \\
2.54 \\
2.47 \\
2.42 \\
2.38 \\
2.31 \\
2.27 \\
2.19 \\
2.08 \\
1.985 \\
1.926 \\
1.873 \\
1.797 \\
1.768 \\
1.734 \\
1.704\end{array}$ & $\begin{array}{r}10 \\
5 \\
8 \\
2 \\
9 \\
7 \\
2 \\
1 \\
4 \\
4 \\
3 \\
4 \\
4 \\
1 \\
1 \\
2 \\
3 \\
4 \\
5 \\
1 \\
6 \\
2 \\
5 \\
1 \\
1 \\
2\end{array}$ & $\begin{array}{l}\text { 1. } 658 \\
1.642 \\
1.610 \\
1.590 \\
1.563 \\
1.533 \\
1.512 \\
1.429 \\
1.382 \\
1.365 \\
1.332 \\
1.313 \\
1.298 \\
1.275 \\
1.248 \\
1.222 \\
1.211 \\
1.202 \\
1.191 \\
1.181 \\
1.168 \\
1.143 \\
1.118 \\
1.094 \\
1.086 \\
1.060\end{array}$ & $\begin{array}{l}1 \\
4 \\
3 \\
1 \\
2 \\
4 \\
1 \\
3 \\
3 \\
3 \\
1 \\
2 \\
1 \\
3 \\
2 \\
1 \\
2 \\
2 \\
1 \\
1 \\
2 \\
2 \\
1 \\
2 \\
2 \\
1\end{array}$ \\
\hline
\end{tabular}

b Broad. bd Broad, diffuse. a Diffuse. 


\section{ZIPPEITE}

\section{Near $2 \mathrm{UO}_{3} \cdot \mathrm{SO}_{3} \cdot 5 \mathrm{H}_{2} \mathrm{O}$}

A number of different basic uranyl sulfates apparently are confused under the name zippeite. The spacing data here given are for a particular type of natural material, that also has been synthesized, to which it is convenient to restrict the name. (See Frondel, 1952.) This mineral occurs at Joachimsthal, Bohemia, and seems to be widely distributed. Data for one of the other zippeite-like minerals, from the Happy Jack mine, Utah, are also given below.

\begin{tabular}{|c|c|c|c|c|c|}
\hline \multicolumn{4}{|c|}{ Joachimsthal } & \multicolumn{2}{|c|}{ Happy Jack mine, Utah } \\
\hline$d(\mathrm{~A})$ & $I$ & $d(\mathrm{~A})$ & $I$ & $d(\mathrm{~A})$ & $I$ \\
\hline $\begin{array}{l}8.65 \\
7.06 \\
6.45 \\
\text { 5. } 44 \\
\text { 4. } 27 \\
\text { 3. } 88 \\
\text { 3. } 63 \\
\text { 3. } 51 \\
\text { 3. } 12 \\
\text { 2. } 86 \\
\text { 2. } 71 \\
\text { 2. } 65 \\
\text { 2. } 47 \\
2.34 \\
2.21 \\
2.14 \\
2.09 \\
2.05 \\
1.935 \\
1.876 \\
1.856 \\
1.816 \\
1.745 \\
1.694 \\
1.666 \\
1.632\end{array}$ & $\begin{array}{r}4 \\
10 \\
2 \\
4 \\
5 \\
3 \\
4 \\
8 \\
9 \\
6 \\
2 \\
5 \\
5 \\
2 \\
4 \\
3 \\
2 \\
5 \\
6 \\
2 \\
2 \\
5 \\
6 \\
1 \\
1 \\
1\end{array}$ & $\begin{array}{l}1.598 \\
1.563 \\
1.519 \\
1.494 \\
1.458 \\
1.424 \\
1.396 \\
1.367 \\
1.326 \\
1.299 \\
1.258 \\
1.236 \\
1.219 \\
1.198 \\
1.180 \\
1.162 \\
1.139 \\
1.106 \\
1.093 \\
1.067 \\
1.056 \\
1.037 \\
1.024 \\
1.011 \\
1.001\end{array}$ & $\begin{array}{l}5 \\
2 \\
2 \\
1 \\
2 \\
4 \\
1 \\
1 \\
1 \\
1 \\
1 \\
1 \\
1 \\
1 \\
1 \\
1 \\
1 \\
2 \\
2 \\
2 \\
1 \\
1 \\
1 \\
1 \\
1 \\
1\end{array}$ & $\begin{array}{l}9.61 \\
7.31 \\
5.57 \\
4.77 \\
\text { 4. } 27 \\
\text { 4. } 10 \\
3.99 \\
3.66 \\
\text { 3. } 51 \\
3.22 \\
3.15 \\
2.86 \\
2.66 \\
2.53 \\
2.45 \\
2.25 \\
2.22 \\
2.12 \\
2.002 \\
1.96 \\
1.884 \\
1.855 \\
1.821 \\
1.791 \\
1.749 \\
1.731 \\
1.701 \\
1.621 \\
1.583 \\
1.517\end{array}$ & $\begin{array}{c}1 \\
10 \\
1 \\
1 \\
1 \\
4 \\
1 \\
9 \\
5 \\
1 \\
8 \\
4 \\
3 \\
4 \\
1 \\
2 \\
2 \\
4 \\
4 \\
2 \text { b } \\
2 \text { b } \\
1 \\
2 \text { b } \\
1 \text { b } \\
4 \\
1 \\
4 \\
2 \\
3 \\
1\end{array}$ \\
\hline
\end{tabular}

b Broad. d Diffuse. 


\section{REFERENCES}

Arnott, R. J., 1950, X-ray diffraction data on some radioactive oxide minerals: Am. Mineralogist, v. 35, p. 386-400.

Axelrod, J. M., Grimaldi, F. S., Milton, C., and Murata, K. J., 1951, The uranium minerals from the Hillside mine, Yavapai County, Ariz.: Am. Mineralogist v. 36, p. 1-22.

Bannister, F. S., and Horne, J. E. T., 1950, A radioactive mineral from Mozambique related to davidite: Mineralog. Mag., v. 29, p. 101-112.

Berman, R., 1956, Studies of uranium minerals-Fully hydrated torbernite and zeunerite: Am. Mineralogist. (In press.)

Bignand, C., 1955, Sur les propriétés et les synthèses de quelques minéraux uranifères: Soc. franç. minéralogie Bull., tome 78, p. 1-26.

Bignand, C., Goñi, J., and Guillemin, C., 1954, La phosphuranylite, ses relations avec la dewindtite et la renardite: Soc. franc. minéralogie Bull., tome 77, p. 1299-1306.

Bowie, S. H. U., and Horne, J. E. T., 1953, Cheralite, a new mineral of the monazite group: Mineralog. Mag., v. 30, p. 93-99.

Brophy, G. P., and Kerr, P. F., 1953, Hydrous uranium molybdate in Marysvale [Utah] ore: U. S. Atomic Energy Comm. RME-3046, p. 45-51.

Donnay, Gabrielle, and Donnay, J. D. H., 1955, Contribution to the crystallography of uranium minerals: U. S. Geol. Survey TEI-507, U. S. Atomic Energy Commission, Tech. Inf. Service, Oak Ridge, Tenn.

Evans, H. T., and Frondel, C., 1950, Studies of uranium minerals-II, Liebigite and uranothallite: Am. Mineralogist, v. 35, p. 251-254.

Frondel, Clifford, 1950a, Studies of uranium minerals-I, Parsonsite and randite: Am. Mineralogist, v. 35, p. 245-250.

1950b, Studies of uranium minerals-V, Phosphuranylite: Am. Mineralogist, v. 35, p. 756-763.

- 1951a, Studies of uranium minerals-IX, Saleeite and novacekite: Am. Mineralogist, v. 36, p. 680-686.

- $1951 \mathrm{~b}$, Studies of uranium minerals-VIII, Sabugalite, an aluminumautunite: Am. Mineralogist, v. 36, p. 671-679.

1952, Studies of uranium minerals-X, Uranopilite: Am. Mineralogist, v. 37 , p. $950-959$.

1953, Hydroxyl substitution in thorite and zircon: Am. Mineralogist, v. 38 , p. $1007-1018$.

1954, Bassetite and uranospathite: Mineralog. Mag., v. 30, p. 343-353.

1956, Studies of uranium minerals-XI, Gummite: Am. Mineralogist. (In press.)

Frondel, Clifford, and Cuttitta, Frank, 1954, Studies of uranium minerals-XIV, Renardite: Am. Mineralogist, v. 39, p. 448-451.

Frondel, Clifford, and Meyrowitz, Robert, 1956, Studies of uranium mineralsXIX, Rutherfordine, diderichite and clarkeite: Am. Mineralogist, v. 41, p. 127-133.

Frondel, J. W., 1951, Studies of uranium minerals-VII, Zeunerite: Am. Mineralogist, v. 36, p. 249-255.

Frondel, J. W., and Cuttitta, F., 1953, Studies of uranium minerals-XII, The status of billietite and becquerelite: Am. Mineralogist, v. 38, p. 1019-1024.

1954, Studies of uranium minerals-XVI, An alteration product of ianthinite: Am. Mineralogist, v. 39. p. 1018-1020.

Gruner, J. W., 1954, The chemical formula of clarkeite: Am. Mineralogist, v. 39 , p. 836-838. 
Hogarth, D. C., and Nuffield, E. W., 1954, Studies of radioactive compoundsVII, Phosphuranylite and dewindtite: Am. Mineralogist, v. 39, p. 444-447.

Hurlbut, C. S., Jr., 1954, Studies of uranium minerals-XV, Schroeckingeritefrom Argentina and Utah: Am. Mineralogist, v. 39, p. 901-907.

Kerr, P. F., and Holland, H. D., 1951, Differential thermal analysis of davidite: Am. Mineralogist, v. 36, p. 563-572.

Milne, I. H., and Nuffield, E. W., 1951, Studies of radioactive compounds-I, Vandenbrandeite: Am. Mineralogist, v. 36, p. 394-410.

Mrose, M. E., 1953, Studies of uranium minerals-XIII, Synthetic uranospinites: Am. Mineralogist, v. 38, p. 1159-1168.

Nuffield, E. W., and Milne, I. H., 1953, Studies of radioactive compounds-VI, Meta-uranocircite: Am. Mineralogist, v. 38, p. 476-488.

Pabst, A, 1951, Huttonite, a new monoclinic thorium silicate: Am. Mineralogist,. v. 36 , p. $60-69$.

1954, Brannerite from California: Am. Mineralogist, v. 39, p. 109-117.

Ross, Virginia, 1955a, Studies of uranium minerals-XXI, Synthetic hydrogenautunite: Am. Mineralogist, v. 40, p. 917-919.

- 1955b, Studies of uranium minerals-XVII, Synthetic schroeckingerite:: Am. Mineralogist, v. 40, p. 515-519.

Stern, T. W., Stieff, L. R., Girhard, M. N., and Meyrowitz, Robert, 1956, The occurrence and properties of metatyuyamunite: Am. Mineralogist, v. 41,. p. 187-201.

Thompson, M. E., Ingram, Blanche, and Gross, E. B., 1956, Abernathyite, a: new uranium mineral of the metatorbernite group: Am. Mineralogist, v. 41, p. 82-90.

Thompson, M. E., Weeks, A. D., and Sherwood, A. M., 1955, Rabbittite, a new: uranyl carbonate from Utah: Am. Mineralogist, v. 40, p. 201-212.

Vaes, J. F., and Kerr, P. F., 1949, Sengierite, a preliminary description: Am.. Mineralogist, v. 34, p. 109-120. 
